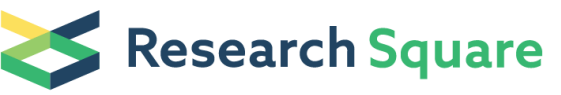

Preprints are preliminary reports that have not undergone peer review.

They should not be considered conclusive, used to inform clinical practice, or referenced by the media as validated information.

\section{Integrated wireless theranostic contact lens for in situ electrical sensing and regulation of glaucoma}

\section{Cheng Yang}

State Key Laboratory of Optoelectronic Materials and Technologies, Sun Yat-Sen University

\section{Qianni Wu}

State Key Laboratory of Ophthalmology, Zhongshan Ophthalmic Center, Sun Yat-Sen University

Junqing Liu

Department of Cardiology, the First Affiliated Hospital of Jinan University

Jingshan Mo

State Key Laboratory of Optoelectronic Materials and Technologies, Sun Yat-Sen University

\section{Xiangling Li}

State Key Laboratory of Optoelectronic Materials and Technologies, Sun Yat-Sen University

\section{Chengduan Yang}

State Key Laboratory of Optoelectronic Materials and Technologies, Sun Yat-Sen University

Ziqi Liu

State Key Laboratory of Optoelectronic Materials and Technologies, Sun Yat-Sen University

Jingbo Yang

State Key Laboratory of Optoelectronic Materials and Technologies, Sun Yat-Sen University

\section{Lelun Jiang}

School of Biomedical Engineering, Sun Yat-Sen University

\section{Weirong Chen}

State Key Laboratory of Ophthalmology, Zhongshan Ophthalmic Center, Sun Yat-Sen University

\section{Hui-jiuan Chen}

State Key Laboratory of Optoelectronic Materials and Technologies, Sun Yat-Sen University

\section{Ji Wang}

The First Affiliated Hospital of Sun Yat-Sen University, Sun Yat-Sen University

\section{Xi Xie ( $\nabla$ xiexi27@mail.sysu.edu.cn )}

State Key Laboratory of Optoelectronic Materials and Technologies, Sun Yat-Sen University https://orcid.org/0000-0001-7406-8444

\section{Article}

Keywords: Wireless theranostic system, Smart contact lens, Glaucoma monitoring and therapy, Closedloop system, Wireless sensing. 
Posted Date: July 13th, 2021

DOl: https://doi.org/10.21203/rs.3.rs-645899/v1

License: (c) (i) This work is licensed under a Creative Commons Attribution 4.0 International License. Read Full License 


\section{Integrated wireless theranostic contact lens for in situ electrical sensing and regulation of glaucoma}

Cheng Yang ${ }^{1,3}$, Qianni $\mathrm{Wu}^{2}$, Junqing Liu ${ }^{4}$, Jingshan $\mathrm{Mo}^{1}$, Xiangling $\mathrm{Li}^{1,5}$, Chengduan Yang $^{1,3}$, Ziqi Liu ${ }^{1}$, Jingbo Yang ${ }^{1,5}$, Lelun Jiang ${ }^{5}$, Weirong Chen ${ }^{2}$, Hui-jiuan Chen ${ }^{1}$, Ji $\mathrm{Wang}^{3}, \mathrm{Xi} \mathrm{Xie^{1,3* }}$

1. State Key Laboratory of Optoelectronic Materials and Technologies, Guangdong Province Key Laboratory of Display Material and Technology, School of Electronics and Information Technology, Sun Yat-Sen University, Guangzhou, 510006, China

2. State Key Laboratory of Ophthalmology, Zhongshan Ophthalmic Center, Sun YatSen University, Guangzhou, 510006, China

3. The First Affiliated Hospital of Sun Yat-Sen University, Sun Yat-Sen University, Guangzhou, 510006, China

4. Department of Cardiology, the First Affiliated Hospital of Jinan University, Guangzhou 510630, China

5. School of Biomedical Engineering, Sun Yat-Sen University, Guangzhou, 510006, China

Email: xiexi27@mail.sysu.edu.cn 
Keywords: Wireless theranostic system, Smart contact lens, Glaucoma monitoring and therapy, Closed-loop system, Wireless sensing.

\begin{abstract}
:
Engineered closed-loop devices that can wirelessly track intraocular pressure (IOP) and offer feedback-medicine administrations are highly desirable for glaucoma treatments, yet remain difficult to develop. Integrated theranostic systems based on contact lens still confront several challenges, including size limits, requirements of wireless operations, and cross-coupling between multiple functional modulus. Here, for the first time to our knowledge, an integrated wireless theranostic contact lens (WTCL) for in situ electrical sensing and on-demand drug delivery of glaucoma was developed. The WTCL utilized a highly compact circuitry and structural design, which enabled highdegreed integration of IOP sensing and electrically controlled delivery modulus on the curved and limited surface of contact lens. The wireless IOP sensing modulus could ultra-sensitively detect IOP fluctuations, due to the unique cantilever configuration design of LCR circuit with ultra-soft air dielectric film sandwiched between each capacitive sensing plate. The drug delivery modulus employed a highly efficient wireless power transfer circuit, to trigger delivery of anti-glaucoma drug into aqueous chamber via iontophoresis to enhance drug permeation across cornea. The specialized design of frequency separation enabled individual operations of different modules without cross-coupling. The noninvasive, smart, wireless and closed-loop theranostic features endowed the WTCL as a highly promising system for glaucoma treatments.
\end{abstract}




\section{Introduction}

Intelligent point-of-care electrical platforms that could provide real-time health assessment and medical intervention would greatly relieve many acute and stubborn diseases $^{1-6}$. Among the diseases, glaucoma can cause irreversible vision loss of patients ${ }^{7}$, which is often deteriorated by the elevation of intraocular pressure (IOP) due to abnormal circulation of aqueous humor ${ }^{7-9}$. Since IOP varies associated with human activities and circadian rhythm ${ }^{10}$, it needs long-term and continuous tracking to analyze the critical IOP fluctuations for identifying optimal therapeutic conditions ${ }^{11,12}$. At present, many types of ophthalmotonometers (e.g. indentation tonometry, applanation tonometry, rebound tonometry, and dynamic contour tonometry) have provided snapshot measurements of IOP for glaucoma diagnosis in hospitals ${ }^{13}$, yet the operations generally required trained clinicians, and failed to collect many critical IOP fluctuation ${ }^{14}$. On the other hand, clinically medicine administrations for glaucoma treatments have been relying on topical drug delivery via eyedrops to reduce IOP for suspend the deterioration of vision that glaucoma caused ${ }^{8,13,15}$. However, conventional drug deliveries into the anterior chamber remain challenging (low intraocular bioavailability, inevitable side-effects, and poor patient adherence) due to the diffusion barriers of cornea ${ }^{16,17}$, and lack the possibility of integration with smart biodevices for on-demand drug delivery. Especially for acute angle-closure glaucoma featured with sudden rise of $\mathrm{IOP}^{18}$, it is usually accompanied by headache, nausea and vomiting that hinders manually self-administrations by patients $^{8}$, while the delayed reduction of IOP will inevitably causes ischemic infarcts and damage optic nerve ${ }^{13}$.

Contact lens, an ideal platform contacted with human eye intimately ${ }^{19,20}$, has been exploited as wearable devices for physiological measurements ${ }^{2,21-24}$. In recent decades, contact lens-based IOP sensors integrated with resonant circuits, microfluidic chips, piezoresistive and photonic crystal technologies have emerged ${ }^{14,21,22,25}$. For example, Park et al. developed colorimetric contact lens for IOP reading based on photonic crystal sensor coupled with microhydraulic strategy to amplify sensitivity ${ }^{22}$. To acquire electrical signals of IOP, Kim et al. demonstrated graphene/Ag nanowires and silicon

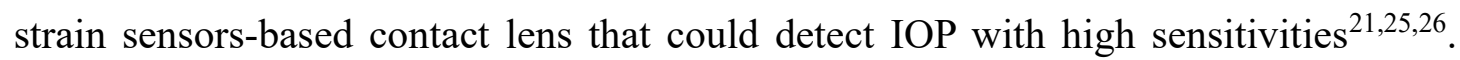


Besides, controlled ocular drug deliveries mediated via contact lens devices have employed versatile strategies including thermal-responsive, enzyme triggering, and hydrogel layer-controlled drug release ${ }^{27-29}$. To reduce burst release of drug from devices, Cakmak et al. fabricated a multi-diffusion layers-based contact lens that could achieve stable ophthalmic drug administration with constant rate ${ }^{29}$. However, medicines permeabilities into aqueous chamber by these passive diffusion methodologies are generally compromised due to the physiological barriers of eye, especially the frequent tear clearance and the tightly packed corneal epithelium cells ${ }^{30}$. While most of the existing strategies for glaucoma applications focus on either sensing or delivery separately, integrated systems for closed-loop IOP monitor and regulation are highly desirable to treat glaucoma, yet remain undeveloped.

Closed-loop theranostic systems on flexible patches have recently been developed to automatically monitor biomarkers, and respond rapidly to treat these complications $\mathrm{s}^{1,3,4,31}$. However, in contrast to patch devices worn on skin, closed-loop theranostic systems based on contact lens confront several complicating challenges due to its nature of limited size and requirement of wireless operations. First, contact lens is flexible, lightweight, curved and ultrathin device with extremely limited area ${ }^{32,33}$, which is highly challenging to install intricate theranostic system composited by multimodules, and less compatible with standard 2D micro/nano-fabrication routes. Second, contact lens devices need to operate wirelessly to promote patients' comforts $^{34}$, yet the potential cross-coupling between wireless sensing and delivery modulus on limited device area would interfere to their individual operations. Third, simultaneous satisfactions of detection sensitivity and on-demand drug delivery on a single device are also difficult, since the limited space of contact lens would restrict the sizes of sensor or delivery module to achieve effective operations. 

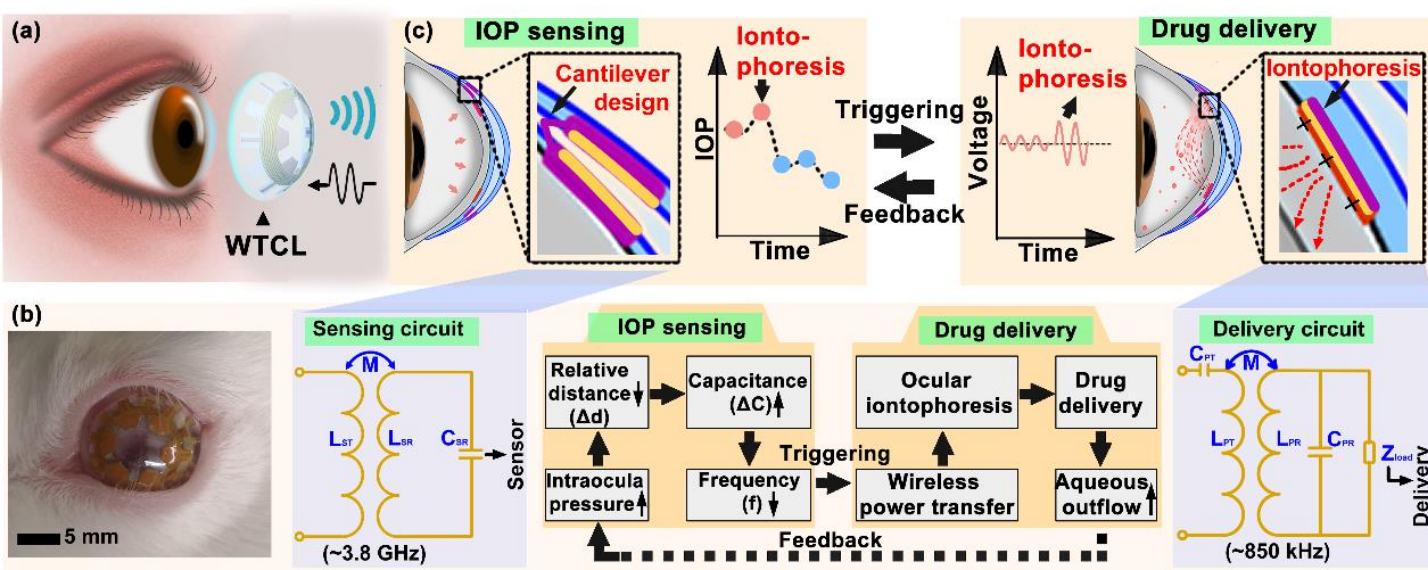

IOP sensing
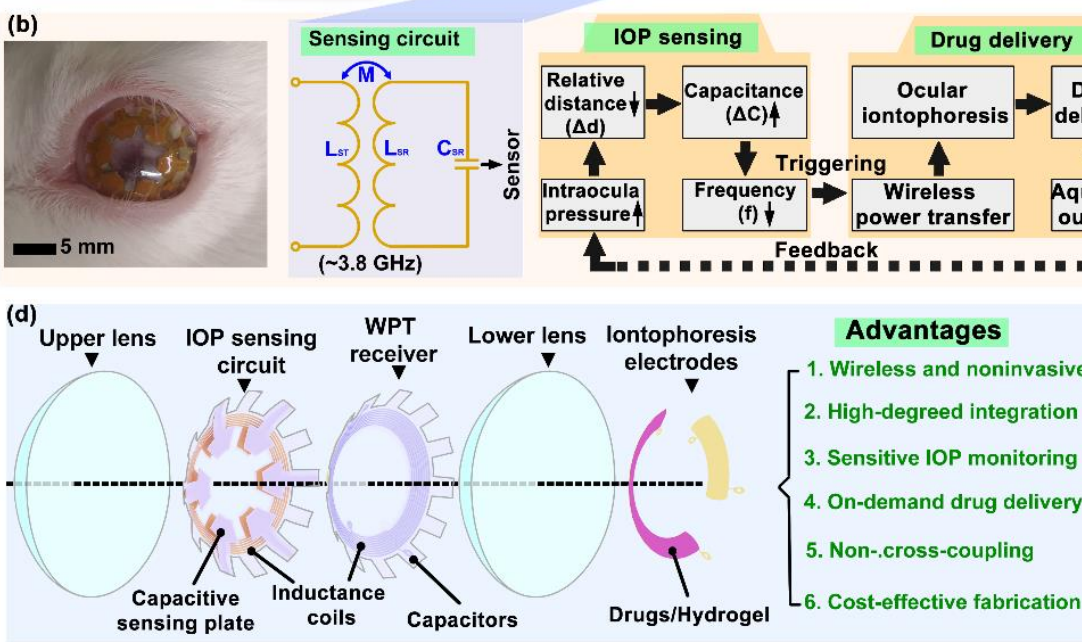

Advantages

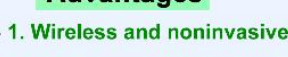

2. High-degreed integration

3. Sensitive IOP monitoring

4. On-demand drug delivery

5. Non-.cross-coupling

6. Cost-effective fabrication

(e)

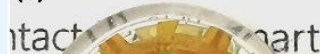

itacto 2 inart

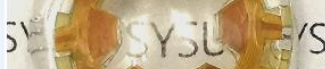

itaclent int

Figure 1. Schematic of the WTCL for real-time and in situ glaucoma diagnosis and

therapy in a closed-loop manner. (a) Schematic of the WTCL for wireless glaucoma diagnosis and therapy. (b) Photograph of WTCL worn on the eyes of a live rabbit. (c) Schematic of wireless operation for the purpose of IOP monitoring and on-demand medicines administration in a closed-loop manner. The soft device, engineered as a double layer contact lens structure, was integrated with an LCR and a WPT receiver circuit. These modules were wirelessly connected to external integrated antenna that could record the IOP signal and trigger iontophoresis for drug delivery if needed. Insert figures respectively highlight critical IOP sensing and drug delivery unit. (d) Structure of the WTCL in an exploded view. (e) Optical image of the WTCL.

In this work, for the first time to our knowledge, an integrated wireless theranostic contact lens (WTCL) was developed, for in situ glaucoma monitoring and electrically triggered drug administration at high-risk IOP conditions (Figure 1a). The WTCL employed a highly compact structural design and circuits layout, which enabled highdegreed integration of IOP sensing and on-demand delivery modulus on the curved and 
limited surface of contact lens without vision blockage (Figure 1b). The IOP sensing modulus possessed a unique cantilever configuration of LCR circuit, where each capacitive sensing plate sandwiching ultra-soft air dielectric film could ultra-sensitively respond to the IOP changes, producing detectable resonant frequency signals for wireless recording. The drug delivery modulus utilized a highly efficient wireless power transfer (WPT) circuit to drive anti-glaucoma drugs coated on electrode surface to migrate into the aqueous chamber via iontophoresis, which offered an electrical switch for drug delivery and enhancement of drug permeation across cornea (Figure 1c). The specialized design of wireless sensor and WPT receiver enabled channel separation via different operational frequencies without cross-coupling, ensuring the individual functions of modulus in an integrated closed-loop system. The noninvasive, smart, wireless and closed-loop theranostic features of the WTCL endowed this platform as a highly promising tool for facilitating glaucoma treatments and preventing acute symptoms.

\section{Results}

\section{System design and fabrications of the WTCL}

Soft contact lens conformally interface with the cornea could effectively deform to transduce the expansion of the corneal limbus to the integrated sensor circuit when IOP increases, and localize external stimulations (e.g. electricity or chemicals) exerted on the cornea. Double layer-lens structure, a typical design acceptable for contact lens devices $^{34,35}$, was adopted for fabricating WTCL to benefit integration of LCR circuit and drug administration module (Figure 1d) on the extremely limited space of contact lens (Figure 1e). The air film sandwiched between two layers of lens combined with LCR circuit characterized by cantilever structure formed the IOP transducer that could detect pressure fluctuations and transmit it wirelessly ${ }^{25,36}$. At high-risk IOP condition $(\mathrm{IOP}>21 \mathrm{mmHg}$ ), WPT triggered iontophoresis enables in situ drug administration effectively. Key advantages of this device contain: 1) soft, lightweight, re-usable, and noninvasive features as well as wireless operations are highly compatible with contact lens platform. 2) Compact structural design and circuits layout enable high-degreed integration of IOP monitoring and on-demand delivery modulus in limited area without 
vision blockage. 3) Rational circuit designs enabled sensitive IOP monitoring by unique cantilever sensor structure, on-demand and effective ocular drug delivery via iontophoresis, individually controlled channel without cross-coupling via frequency separation. 4) The closed-loop system with entirely electrical interface is beneficial for signal collection, processing, feedback, and transmission, as well as programmable ondemand drug administration. 5) Fabrication of the device is compatible with existing large-scale and cost-effective manufacturing process, emphasizing its potential for widespread applications.

The IOP monitoring circuit employed a unique snowflake-shaped layout design (Figure 2a), where each capacitive sensing plate (totally 6 plates) was then aligned with reference plate (Figure 2b) by folding to form a cantilever configuration (Figure 2c). The reference plates and 5 coils of inductance were embedded in the upper lens (Figure 2d), while dangling sensing plates contacting the front surface of the lower lens, with a dielectric air film between the reference and sensing plates forming a variable capacitor (Figure S1.1). The capacitance combined with inductance coil formed a LCR circuit for wirelessly IOP monitoring. The deformation of corneal curvature caused by increased IOP compresses the thickness of air dielectric layer $(\Delta d)$, leading to the rise of capacitance $\left(\mathrm{C}_{\mathrm{SR}}\right)$ and reduction of resonant frequency of LCR circuit that could be recorded by reading coil of integrated antenna (Figure 2e) wirelessly ${ }^{25,36}$. Due to the ultra-soft (ultra-low elastic modulus and zero viscoelasticity) feature of the sandwiched air film, the variable capacitors formed by cantilever configuration can ultra-sensitively respond according to the change of pressure. The cantilever design effectively avoid the issues of redundant serial capacitors, and complicated device fabrication process especially the wire bonding step that have been encountered in previous reported strategies $^{36,37}$. On the other hand, the drug delivery circuit utilized a flower-shaped layout design (Figure 2f) that enables robust interlocking mechanically between the flexible circuit and the lower layer of contact lens (Figure $\mathbf{2 g})^{38}$. The front side of circuit embedded in the lower lens possessed coils (Figure 2f-IV and Figure S1.1) connected with chip capacitor for wireless power harvest, while the drugs-coated iontophoretic electrodes on the bottom side of delivery circuit were exposed and would be in contact 
with the cornea. Anti-glaucoma drugs, brimonidine, was loaded in a hydrogel layer coated on the iontophoretic electrode, which could be delivered into the aqueous humor via wirelessly iontophoresis to reduce IOP. The iontophoresis not only offered nonmechanical switch for drug delivery in a low-power consummation manner, but also facilitate drug penetration across cornea via electrophoresis effects ${ }^{39}$. The rational design of wireless sensor and WPT receiver at different operational frequencies $(\sim 3.8$ $\mathrm{GHz}$ and $\sim 850 \mathrm{kHz}$, respectively) enabled channel separation for individual function. The double layer lens design enabled a compact structure to accommodate multiple electronic modulus positioned in the rim region of the contact lens, hence possessed an open vision window larger than the pupil's size without blocking the views of wearers. The fabrication of the sensing and delivery modulus employed a printed circuit process coupled with cast-molding method. For the sensing module, $\mathrm{Cu}(\sim 100 \mu \mathrm{m})$ was electrodeposited on a flexible polyimide (PI) substrate and patterned via photolithography and wet etching, followed by covering with $\mathrm{Ni} / \mathrm{Au}$ to improve biocompatibility of electrodes. The flexible substrate was cut by laser according to the snowflake-shaped circuit design, folded, and embedded into the upper polydimethylsiloxane (PDMS) lens via cast-molding technique (Figure S1.2), where each folded sensing plate was detached from the contact lens to form a cantilever configuration (Figure $\mathbf{2 h}$ ). For the delivery module, similar to the sensing module, $\mathrm{Cu}$ was electro-deposited on PI substrate and patterned as coils features via photolithography and wet etching to form the WPT receiver. A second layer of PI was spinning coated on top of the coils, and another $\mathrm{Cu}$ layer was prepared according to the iontophoretic electrode pattern, which was connected to the coils at the bottom side by through-holes. The electrodes were further covered with $\mathrm{Ni} / \mathrm{Au}$, and capacitors were soldered onto the front side of circuit to tune the WPT operation frequency, followed by embedding the circuit into the lower PDMS lens via cast-molding. The delivery electrode of lower lens was coated with a thin layer of drugs-loaded hydrogel, and assembled with the upper lens to form the final WTCL (Figure 2i). The compact layout and double layer lens design enables sensors and WPT receiver to be embedded inside contact lens, avoiding direct contact of these 
components to the ocular surface that might cause potential irritations to eye. The WTCL fabrication is compatible with commercial printed circuit board process, indicating the potentials for large-scale manufactures of this biomedical device. For experiments, an integrated antenna (Figure 2e) consisting of concentrically aligned IOP reading coils and WPT transmitter soldered on a matching circuit board was fabricated, which could collect the output signals from the wireless sensor and transfer power to the WPT receiver of the WTCL.
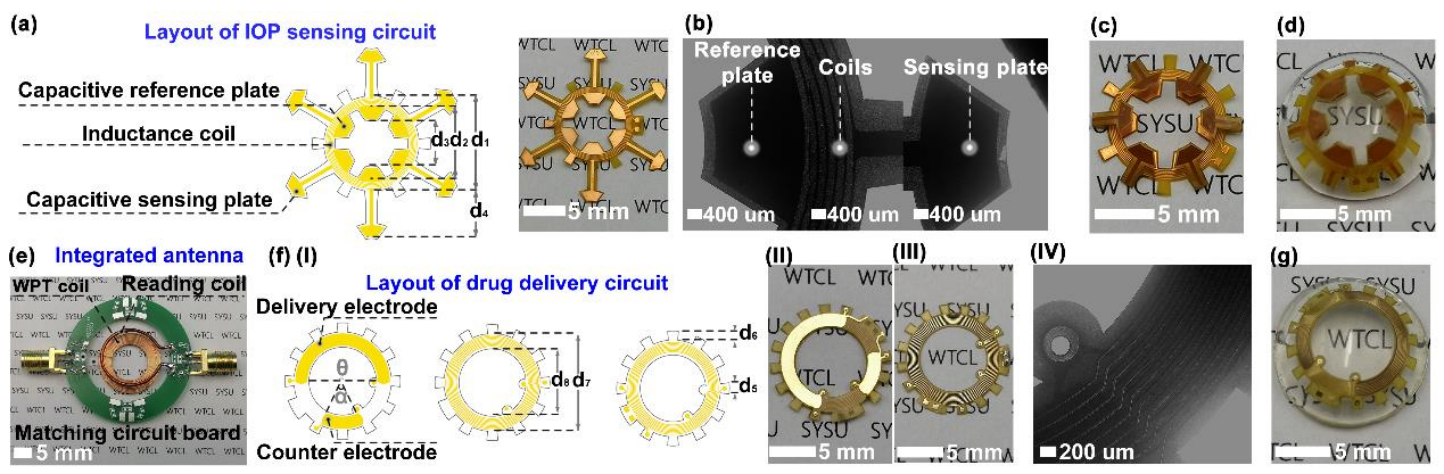

(h) IOP monitoring circuit
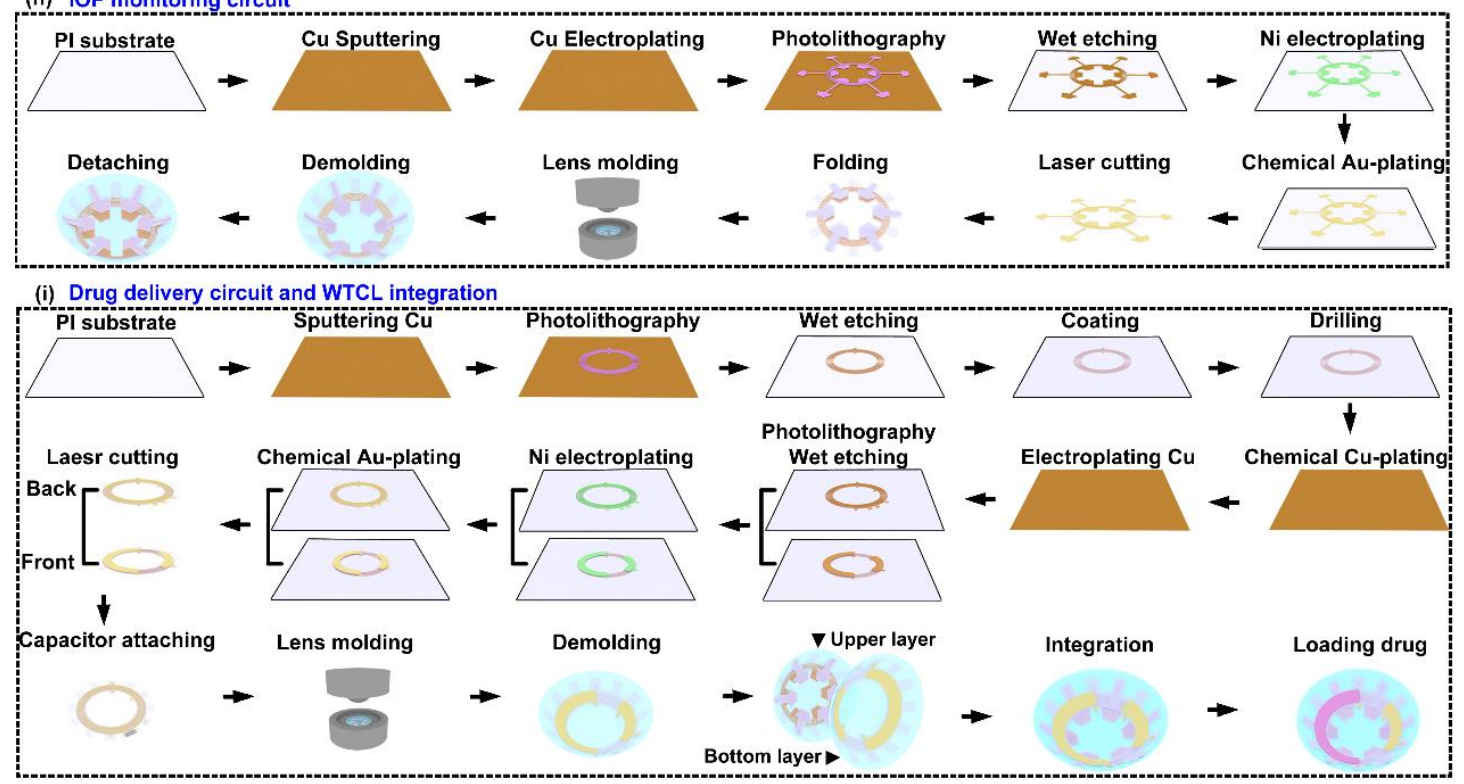

Figure 2. Schematic illustration of the WTCL's design and fabrication process. (a) The snowflake-shaped layout design and the photograph of the sensing circuit. (b) The microscopic image of the reference plate, coils, and sensing plate deployed on sensing circuit. The photograph of (c) the folded sensing circuit and (d) the upper layer of contact lens. (e) Image of the integrated antenna. (f)(I) The flower-shaped layout design, (II) back surface (III) front surface images, and (IV) microscopic image of the drug 
delivery circuit. (g) The photograph of bottom layer lens integrated with drug delivery circuit. Illustration of the fabrication process of (h) IOP monitoring circuit, (i) drug delivery circuit and the device integration. The fabrication of the sensing and delivery modulus employed a printed circuit process coupled with cast-molding method.

\section{In-vitro performance of wireless IOP monitoring}

The sensing performance of the WTCL was tested in vitro using porcine eyeballs, where porcine eyeballs' features similar with human eyeball have been widely employed in many physiological experiments. The IOP in porcine eye was tuned by controlled infusion of saline solution into the anterior chamber via microinfusion pump, with a pressure gauge to monitor the reference IOP. The IOP reading coil (diameter: $17 \mathrm{~mm}$, turns: 1) of the integrated antenna connected to a network analyzer was positioned on top of the WTCL to monitoring the resonance frequency (Figure 3a). The static sensing performance was conducted by stepwise increase of IOP, while the resonance frequency of WTCL at each IOP condition was recorded. The reflection spectra of six representative WTCL devices worn on the porcine eyeball at different IOP $(5-50 \mathrm{mmHg})$ were recorded and analyzed (Figure $3 \mathrm{~b}$ and Figure S2.1a), where the resonant frequency of IOP monitoring module was found to shift to the lower frequency at higher IOP. The return loss (S11) values at different frequencies and IOP conditions were plotted as heatmap diagrams, where the S11 value exhibited a linear pattern in the frequency-IOP heatmap (Figure 3c). The relation of resonance frequency and IOP of each device was analyzed (Figure 3d and Figure S2.2), which was revealed to be in an inversely linear profile (average R-Square $=0.976 \pm 0.015$ ). Theoretically the resonant frequency is in inversely related to the capacitance according to the LCR circuit equation $\mathrm{f}=(2 \pi \sqrt{\mathrm{LC}})^{-1}$. Our results were consistent with the theoretical prediction in that the increase of IOP would reduce the distance between the capacitance electrodes, hence led to the elevation of capacitance value (Figure S2.1b) and reduction of resonant frequency. Noted that the linear relations of all the six devices overlapped well 
(normalized slope variation $<15 \%$ ) with each other (Figure S2.3), indicating the reliability and repeatability of the fabricated device using our design.

A universal standard curve between resonant frequency and IOP was established by averaging all the six linear curves obtained from the measurements on the six representative devices (Figure 3e), where this standard curve could be employed to calculate detected IOP based on measured resonant frequency. The results suggested that the IOP sensors in the WTCL possessed sufficient sensitivity of $1.28 \pm 0.09$ $\mathrm{MHz} / \mathrm{mmHg}$, which was superior or comparable to other wireless IOP sensors (Table $\mathrm{S} 2$ ). This is likely due to the specific cantilever design of sensor, where the ultra-soft air film sandwiched between the sensing capacitive plates is highly mobile, so that the variable capacitors formed by cantilever configuration can respond to the change of pressure in a highly sensitive manner. The linear range of WTCL was wider than 5-50 $\mathrm{mmHg}$, which were desirable for glaucoma monitoring applications. The measured IOP values by the six WTCL devices were derived from the recorded values of resonant frequency, and compared to the reference IOP measured by pressure gauge for analyzing the sensor's static accuracy via error grid analysis (Figure $3 f$ ). The percentage of data points at different error range was quantified, where $>50 \%$ recording was found to be within error $<10 \%$, and $>75 \%$ recording was found to be within error $<20 \%$. The continuous recording of IOP via WTCL was also examined by measuring the resonant frequency and reference IOP via pressure gauge, respectively, where saline solution was injected into anterior chamber at $\mathrm{t}=0 \mathrm{~s}$ and $883 \mathrm{~s}$ intending to induce IOP spikes (Figure $3 \mathrm{~g}$ ). The measured resonant frequency (Figure S2.4) was calculated into IOP according to the WTCL's averaged standard curve, and the results were calibrated (Figure S2.5). Considering the possible batch variations of devices and porcine eyeballs in experiments, the detected IOP results via WTCL were calibrated by a pressure gaugemeasured data point at $\mathrm{t}=0 \mathrm{~s}$ (indicated with blue star in Figure $3 \mathrm{~h}$ ). The injections of saline induced rising of IOP, followed by slight IOP declines potentially due to the gradual leakage of solution from eyeball, which were all consistently recorded by both WTCL and pressure gauge. The dynamic recording accuracy of WTCL at each time point was analyzed (Figure 3i) and plotted via an error grid analysis (Figure 3j), and the 
average error was found to be $16.49 \pm 7.58 \%$ with all the errors below $30 \%$. The above results demonstrated the WTCL possessed sufficient sensitivity, linear region and reliability that were desirable for glaucoma monitoring.
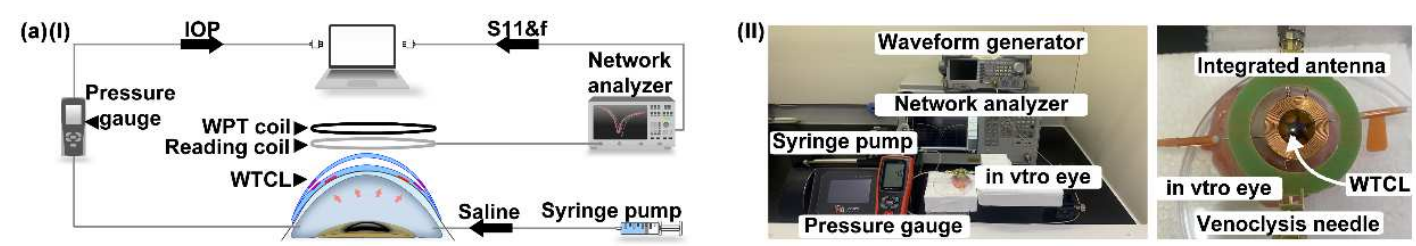

(b) $\quad 5.0 \mathrm{mmHg} \quad 10.0 \mathrm{mmHg} \quad 15.0 \mathrm{mmHg} \quad 20.0 \mathrm{mmHg} \quad 25.0 \mathrm{mmHg} \quad 30.0 \mathrm{mmHg} \quad 35.0 \mathrm{mmHg} \quad 40.0 \mathrm{mmHg} \quad 45.0 \mathrm{mmHg} \quad 50.0 \mathrm{mmHg}$
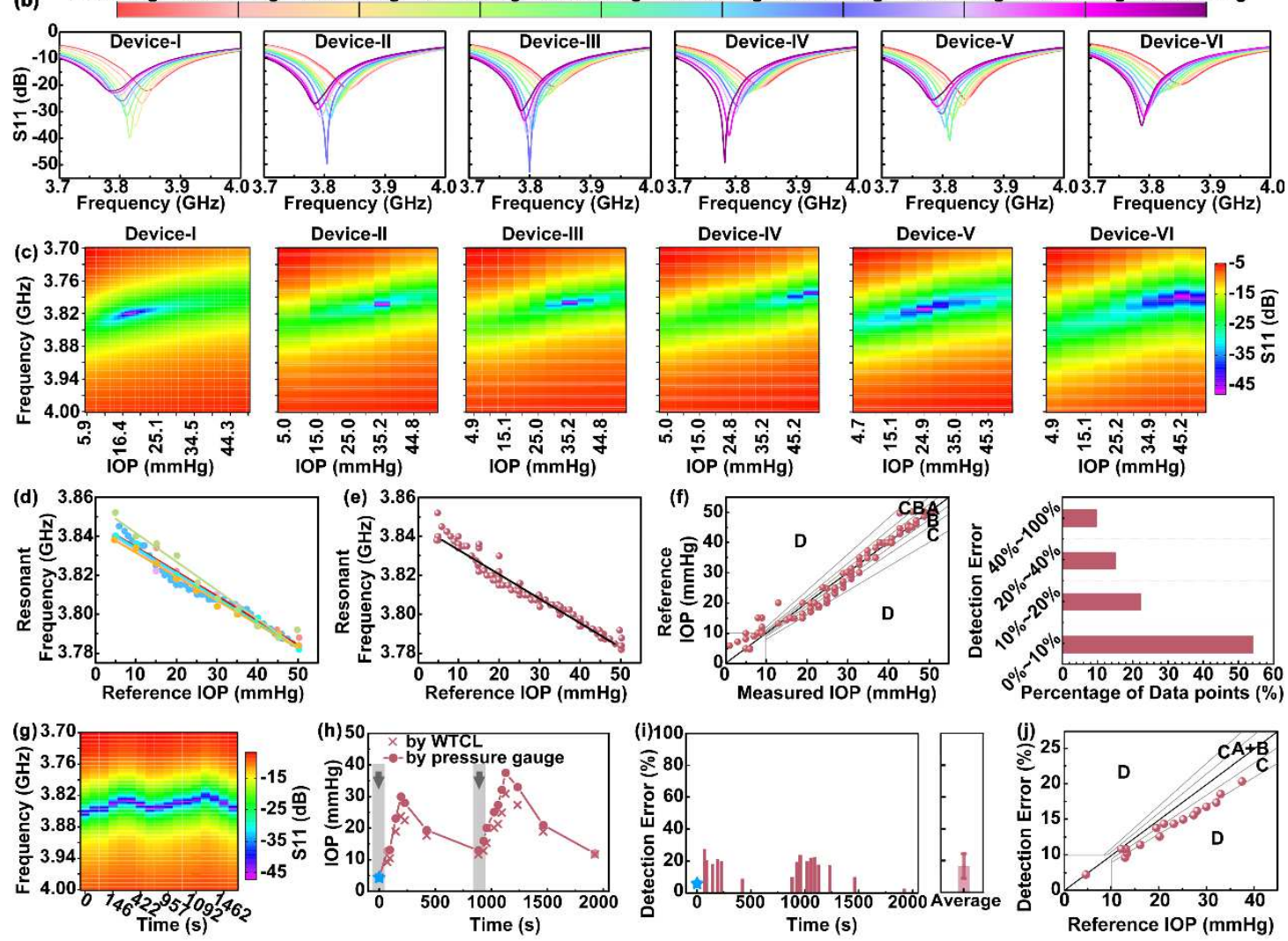

Figure 3. IOP sensing performance of the WTCL. (a-I) Schematic and (II) experimental set-up of the wireless IOP sensing experiments. (b) The reflection spectra of six representative WTCL devices worn on porcine eyeball at different IOP. (c) The results of the S11 values at different frequencies and IOP conditions in (b) were plotted as heatmap diagram, where the value of S11 exhibited a linear pattern in the frequencyIOP heatmap. (d) Linear regression of resonant frequency versus IOP value of each WTCL device. (e) The averaged linear regression of resonant frequency versus IOP value of the six WTCL devices in (b). (f) Error grid analysis and statical analysis of the IOP sensing accuracy via WTCL. Region A, B, C and D referred to errors $<10 \%, 10$ - 
$20 \%, 20-40 \%$ and $>40 \%$, respectively. (g) Heatmap plot of the reflection coefficients recorded during the continuous recording of IOP via WTCL. (h) Continuous IOP signals monitored by WTCL on in-vitro porcine eyeball. Calibration point using reference IOP was marked with blue asterisks. The black arrow referred to the time point of saline injections. (i) Statistical analysis of detection errors via WTCL compared to commercial pressure gauge at different time points. Calibration point was marked with blue asterisks. (j) Error grid analysis of the continuous IOP sensing via WTCL. Region $\mathrm{A}+\mathrm{B}, \mathrm{C}$ and $\mathrm{D}$ referred to errors $<20 \%, 20-40 \%$ and $>40 \%$, respectively.

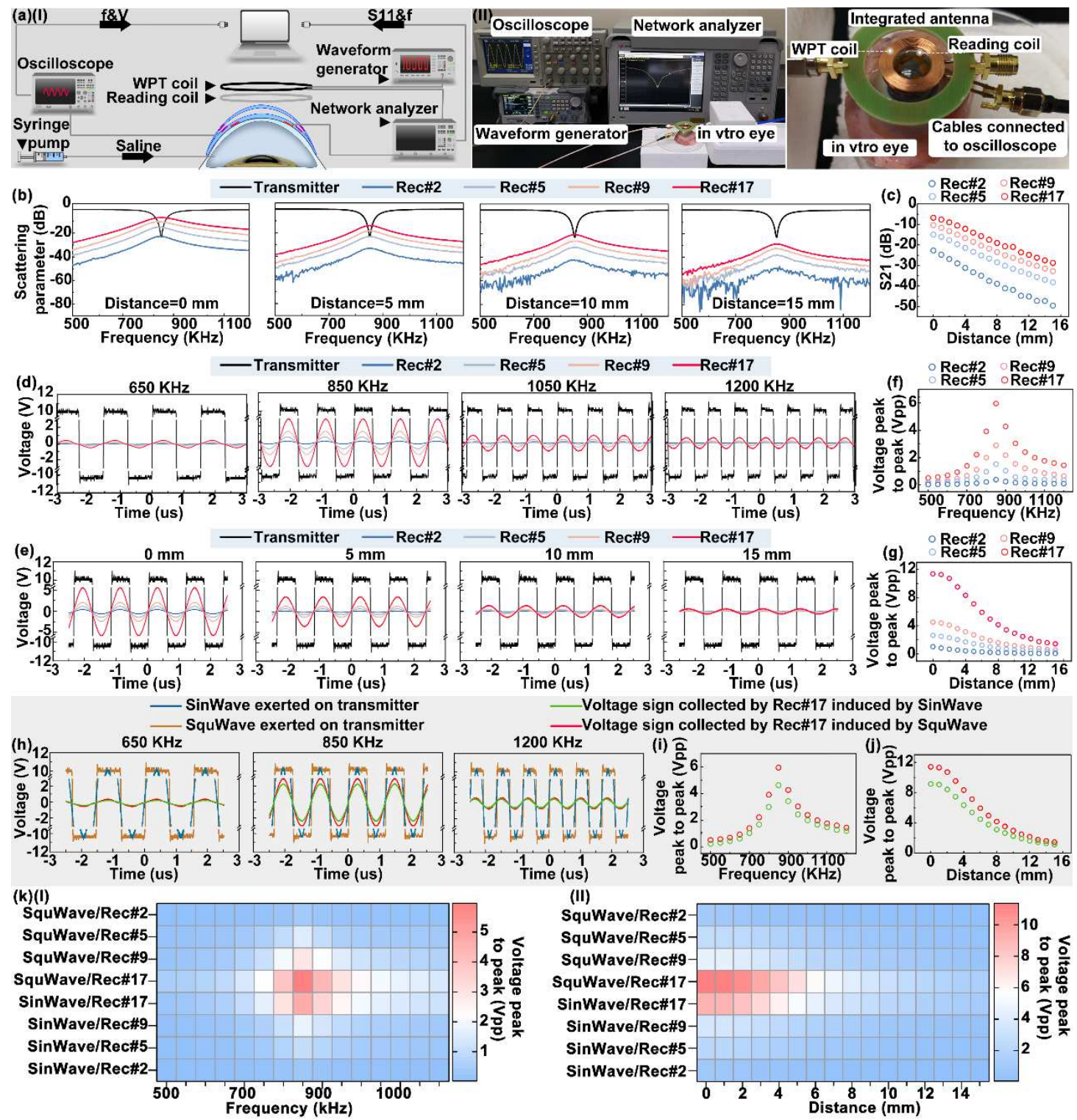

Figure 4. WPT performance of the WTCL. (a-I) Schematic and (II) experimental 
set-up of the WPT experiments. (b) Reflection coefficient spectra (S11 and S21) recorded from four receivers at different radiation distance. (c) The S21 recorded by the four receivers at $850 \mathrm{kHz}$ were plotted as a function of radiation distance. (d) Alternating voltage signals collected from four receivers wirelessly under different frequency radiation at $20 \mathrm{Vpp}$ applied on transmitter, and (e) Wirelessly transferred alternating voltage waveforms of four receivers under different radiation distance at 20 Vpp applied on transmitter, and (f) the Vpp were plotted as a function of frequency and (g) as a function of distance. (h) The wirelessly transferred voltage signals collected by Rec\#17 activated by SquWave or SinWave voltages at different frequencies, and the Vpp were plotted as a function of (i)frequency or (j) distance. (k) Heatmap plot summarized the Vpp recorded from four receive circuits under different voltage transfer conditions, including the coupling frequency, the radiation distance, and the waveforms.

\section{The studies on WPT, cross-coupling and drug delivery}

Magnetic resonance coupling-based WPT has been a competing technique for wireless bioelectronics due to its relatively high power transfer efficiency and resistance to environmental inference ${ }^{40}$. To achieve optimal coupling performance, four types of WPT receivers with 2, 5, 9, and 17 coils-design (namely Rec\#2, Rec\#5, Rec\#9, and Rec\#17, respectively) were designed with other circuit parameters accordingly modified (Figure S3.1 and Table S3). In order to evaluate the power transfer performance, the optimal coupling frequency and acceptable radiation distance between WPT receiver and transmitter were examined. During experiments, the WPT transmitter of the integrated antenna connected to a waveform generator and a network analyzer was aligned over the WTCL with identical axis (Figure 4a), while the WPT receivers were connected to an oscilloscope to monitored the generated voltages. The Reflection coefficient spectra from four receivers at different radiation distance were recorded (Figure 4b and Figure S3.2), where the resonant frequency of transmitter and all receivers were observed to be at $\sim 850 \mathrm{kHz}$ according to the circuit designs. The channel separation between IOP monitoring $(\sim 3.8 \mathrm{GHz})$ and WPT $(\sim 850 \mathrm{kHz})$ was sufficiently large to avoid cross-coupling, which might prevent unexpected activation 
of a nontargeted wireless channel in the closed-loop glaucoma diagnosis and treatment ${ }^{41}$. The return loss (S11) reveals that most of the energy carried by electromagnetic wave could be radiated rather than dissipated in the frequency range of $837.38 \mathrm{kHz}$ to $867.45 \mathrm{kHz}$ ), with a bandwidth of transmitter about $30 \mathrm{kHz}^{42}$. The S21 under $850 \mathrm{kHz}$ of all receivers decreased linearly with the increase of radiation distance (Figure 4c). Considering that certain distance between transmitting coils and contact lens is required to avoid interference to human eyes in practical applications, $6 \mathrm{~mm}$ was chosen as optimal distance between transmitting coils and WTCL in experiments. Sequentially, a series of square wave $(20 \mathrm{Vpp})$ with different frequencies $(500 \mathrm{kHz}$ to $1200 \mathrm{kH}, 50 \mathrm{kHz}$ step) or different distances (0 mm to $15 \mathrm{~mm}, 1 \mathrm{~mm}$ step) were wirelessly exerted on the transmitter, to further verify the optimized coupling frequency and distance. The generated sinusoidal voltages on the receivers were recorded by oscilloscope (Figure 4d, Figure 4e and Figure S3.3-3.4), and the relations between peak to peak (Vpp) values and frequencies were analyzed. At the set distance of $6 \mathrm{~mm}$, the Vpp increased sharply from 500 to $850 \mathrm{kHz}$, and dramatically decreased from $850 \mathrm{kHz}$ to $1.2 \mathrm{MHz}$, where the coupling at $850 \mathrm{kHz}$ displayed maximum $\mathrm{Vpp}$ of $\sim 6 \mathrm{~V}$ (Figure 4f), consisting with the previous results of resonant frequency at $\sim 850 \mathrm{kHz}$. On the other hand, at the set frequency of $850 \mathrm{kHz}$, the Vpp of all receivers decreased with the increase of radiation distance (Figure 4g). The insert loss (S21) and Vpp of Rec\#17 were both significantly higher than those of other receivers at identical conditions, suggesting that the Rec\#17 possessed better matching with the WPT transmitter.

Square wave (SquWave) and sine wave (SinWave), representing common voltage signals in analog electronics, possess distinct characteristics in rising and falling edges. In our experiments, SquWave and SinWave voltage signals featured with $20 \mathrm{Vpp}$ and different frequencies (500 kHz to $1200 \mathrm{kHz}$, with step of $50 \mathrm{kHz}$ ) were exerted on WPT transmitter (Figure $4 \mathrm{~h}$ and Figure S3.5-3.6) at different set distances ( $0 \mathrm{~mm}$ to $15 \mathrm{~mm}$, $1 \mathrm{~mm}$ step) to the Rec\#17 which was chosen as the optimized receiver design. The correspondingly collected $\mathrm{Vpp}$ of receiver showed that the voltage transfer behaviors at SinWave voltage were similar to that at SquWave, where $850 \mathrm{kHz}$ was close to the optimal frequency. Moreover, the Vpp induced by the SquWave voltage wave was 
slightly higher than the that by SinWave (Figure $4 i$ and Figure $4 j$ ), likely due to the fact that SquWave signals with more steeper edges created more rapidly changed magnetic field that is more favorable for WPT performance, compared to the SinWave at identical conditions.

To comprehensively evaluate the optimal conditions of WPT, receiver designs and the voltage transfer conditions (the coupling frequency, the radiation distance, and the waveforms) were systematically analyzed (Figure S3.3-3.8) and summarized in two heatmap diagram (Figure 4k). Although the WPT efficiency was higher at shorter radiation distance, $6 \mathrm{~mm}$ was selected as optimal distance between transmitting coils and WTCL since the contact lens needs certain separation from the transmitting coils in practical applications. The maximum transferred Vpp was observed on the optimal receiver Rec\#17 at the applied SquWave with frequency of $850 \mathrm{kHz}$, which were identified as the optimal conditions for the final WTCL.

The WPT performance of the WTCL was further theoretically analyzed, where the mutual inductance (M), power transfer efficiency $(\eta)$ (Figure S4.1), and the skin effects (Figure S4.2) were calculated based on the circuit design. The Mutual inductance (M), a key factor in the technology of WPT, determines voltage in the coil of receiver circuits, were derived from the magnetic coupling coefficient according to the circuit design of four receivers ${ }^{43}$. The mutual inductance was inversely proportional to the radiation distance between transmitter and receiver circuits, where the Rec\#17 group (transmitter and Rec\#17) exhibited the highest value of mutual inductance than other groups (Figure 5a). The power transfer efficiency of Rec\#17 was further calculated to be $48.4 \%$ at the resonate frequency of $850 \mathrm{kHz}$ and $6 \mathrm{~mm}$ radiation distance, significantly higher than other three receiver designs (Figure 5b), also consisting with the experimental results. Rader chart (Figure 5c) visually summarized performance (S21, Vpp, M, and $\eta$ ) of etch WPT group (Rec\#2, Rec\#5, Rec\#9 or Rec\#17 linked to transmitter with 6 mm radiation distance and $850 \mathrm{kHz}$ ), where Rec\#17 group showing grater area in the chart compared to other alternatives. It demonstrated that Rec\#17 group could be served as optimal power transfer platform for further iontophoretic drug administration in this work. 
The cross-coupling between multiple wireless channels is a significant concern since it may disturb the independent control over the in situ sensing and delivery modulus (Figure 5d). Conventional strategy to spatially avoid cross-coupling is less compatible with contact lens devices due to their limited space for spatially separation of channels ${ }^{44}$. Here we employed a specialized technique of radio frequency separation to solve the cross-coupling issue, based on a compact design of device to accommodate distinguished wireless circuits on the limited area of contact lens. Firstly, the IOP reading coil and WPT transmitter were coupled with the WPT receiver (using Rec\#17) at a set distance of $6 \mathrm{~mm}$, respectively, and the S21 indicating the coupling efficiency and the generated voltages on receiver were separately measured. The coupling between WPT transmitter and receiver exhibited S21 higher than $-40 \mathrm{~dB}$ and reached its maximum value $(-15.6 \mathrm{~dB})$ at around $850 \mathrm{kHz}$, and apparent sinusoidal voltage waveform with $6 \mathrm{Vpp}$ was recorded. The coupling between reading coil and WPT receiver displayed ultra-low $\mathrm{S} 21(<-60 \mathrm{~dB})$, and generated negligible voltage that was close to blank group of uncoupled receiver, suggested cross-coupling between IOP reading coil and WPT receiver rarely occurred.

The WTCL was placed on porcine eye at different IOP $(0-50 \mathrm{mmHg})$, and the reading signals were recorded with or without the presence of radiation from WPT transmitter. The S11-freqency spectra appeared to be overlapping well disregard of the presence of WPT radiation, where a typical example at $30 \mathrm{mmHg}$ IOP was shown in Figure5f-I. The resonance frequency and the peak S11 at different IOP were quantitatively analyzed (Figure5f-II), where the radiation of WPT transmitter did not significantly influent the IOP monitoring, indicating cross-coupling between IOP sensor and WPT transmitter was negligible. 

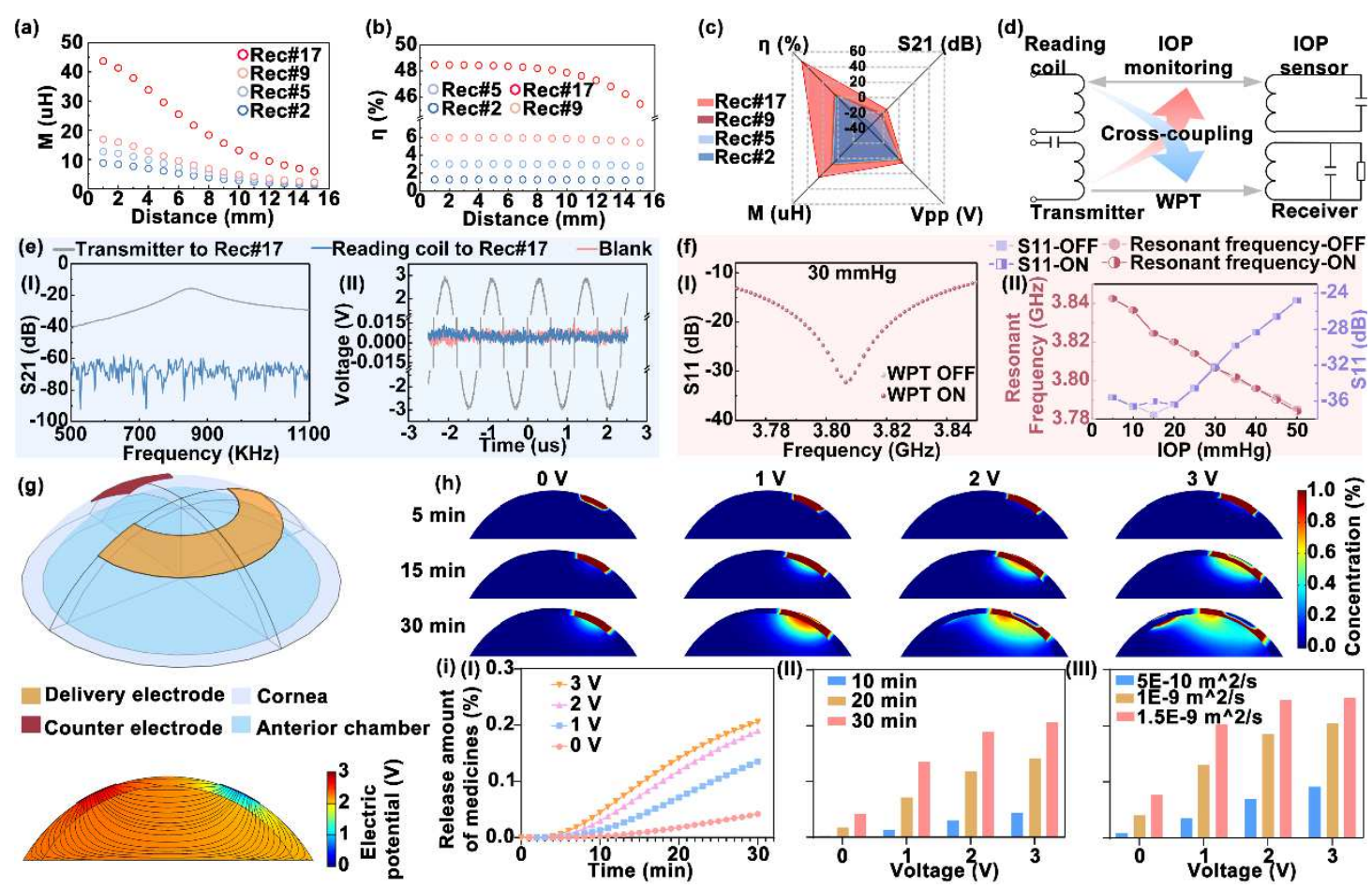

Figure 5. (a) The mutual inductance and (b) Power transfer efficiency were theoretically calculated according to the circuit design of four receivers and the radiation distance. (c) Rader chart summarized performance (S21, Vpp, M, and $\eta$ ) of etch WPT group (Rec\#2, Rec\#5, Rec\#9 or Rec\#17 linked to transmitter with $6 \mathrm{~mm}$ radiation distance and $850 \mathrm{kHz}$ ) (d) Schematic showing the experiments studying the cross-coupling between IOP monitoring and WPT module. Red arrow denoted the interference generated by the radiation of WPT transmitter to sensing module. Blue arrow denotes the cross-coupling between IOP reading coil and the WPT receiver. All examinations were performed with the radiation distance of $6 \mathrm{~mm}$. (e) The IOP reading coil and WPT transmitter were coupled with the WPT receiver (using Rec\#17), respectively, and the S21 indicating the coupling efficiency and the generated voltages on receiver radiated at $850 \mathrm{kHz}$ were separately measured. (f) The WTCL was placed on porcine eye at different IOP, and the reading signals (the resonance frequency and the S11) were recorded with or without the presence of radiation from WPT transmitter. (g) The (top) 3D COMSOL model and (bottom) the simulated distribution profile of electric potential and electric field through the anterior region under the condition of iontophoresis at 3 $\mathrm{V}$ for $\mathrm{t}=30 \mathrm{~min}$. (h) The time slots of drug concentration profile delivered by WTCL at various applied voltages $(0,1,2$, and $3 \mathrm{~V})$. (i) The delivered amounts of drugs at 
different conditions, including the (I) applied voltages, (II) iontophoretic duration and (III) assumed diffusivities, were systematically evaluated.

Brimonidine, maintaining positive charge, is a medication that has been topically delivered in aqueous humor or ciliary body clinically to treat glaucoma by increasing uveoscleral outflow and reducing aqueous fluid production ${ }^{8,15}$. However, corneal barrier comprised of tightly packed epithelium and hydrophilic-hydrophobic interfaces could significantly hinder the passive diffusion of drug molecules from ocular surface into anterior chamber ${ }^{39,45}$. Iontophoresis that drives the migration of charged species via electric field have been successful on transdermal pharmaceuticals delivery ${ }^{46,47}$, and the delivery dose can be tuned by the iontophoretic strength or duration ${ }^{48}$. To achieve effective and controllable ocular drug delivery, iontophoresis is coupled to the WTCL to enhance the transport of bromonidine across the cornea layer in an on-demand manner. Mixture solution of HEMA monomers, crosslinker, photoinitiator and brimonidine tartrate was drop-casted on the iontophoretic electrode surface, and was irradiated with UVB light to form a brimonidine-loaded pHEMA hydrogel layer ${ }^{49}$. When wirelessly powered by the transmitter, the WPT receiver generated alternating voltages on the electrode, which electrically drove the brimonidine into anterior chamber $^{39}$.

To understand the process of iontophoretic delivery, a simplified 3D model (top of Figure 5g and Figure S5.1) imitating the actual scenario of WTCL worn on an eye was established using COMSOL Multiphysics 5.5, where the electric currents interface and the transport of diluted species interface were employed to calculate the electricallydriven drug diffusion profile. The anterior was modeled as a cornea layer (consisting of epithelial cell layer, stroma and epithelial cell layer) covered on anterior chamber, with corresponding electric conductivities and mass diffusivities. A working electrode coated with a thin layer of drug-loaded hydrogel and a counter electrode were conformally placed on the eye, and constant voltage was applied instead of alternating voltage in order to simplify the dynamic simulation process. Detailed boundary condition and parameters were shown in Figure S5.2, Figure S5.3 and Table S4 in 
supplementary materials. The distribution of electric potential and electric field through the anterior region under iontophoresis at $3 \mathrm{~V}$ at $\mathrm{t}=30 \mathrm{~min}$ were shown in bottom of Figure 5g, and the time slots of drug concentration profile at various applied voltages $(0,1,2$, and $3 \mathrm{~V})$ were shown in Figure $5 \mathrm{~h}$ and Figure S5.4. Under iontophoresis, positively charged drug molecule migrated into the anterior chamber, where the drug delivery via iontophoresis was more effective ( $\sim 3$ to 5 -folds higher) than the passive diffusion, due to difficulty of drug diffusion across cornea. The delivered amounts of drugs at different conditions, including the applied voltages, iontophoretic duration and assumed diffusivities, were systematically evaluated (Figure 5i), and the results indicated the increase of these parameters would effectively enhance the drug delivery efficiency.

\section{In vivo performance of the integrated WTCL}



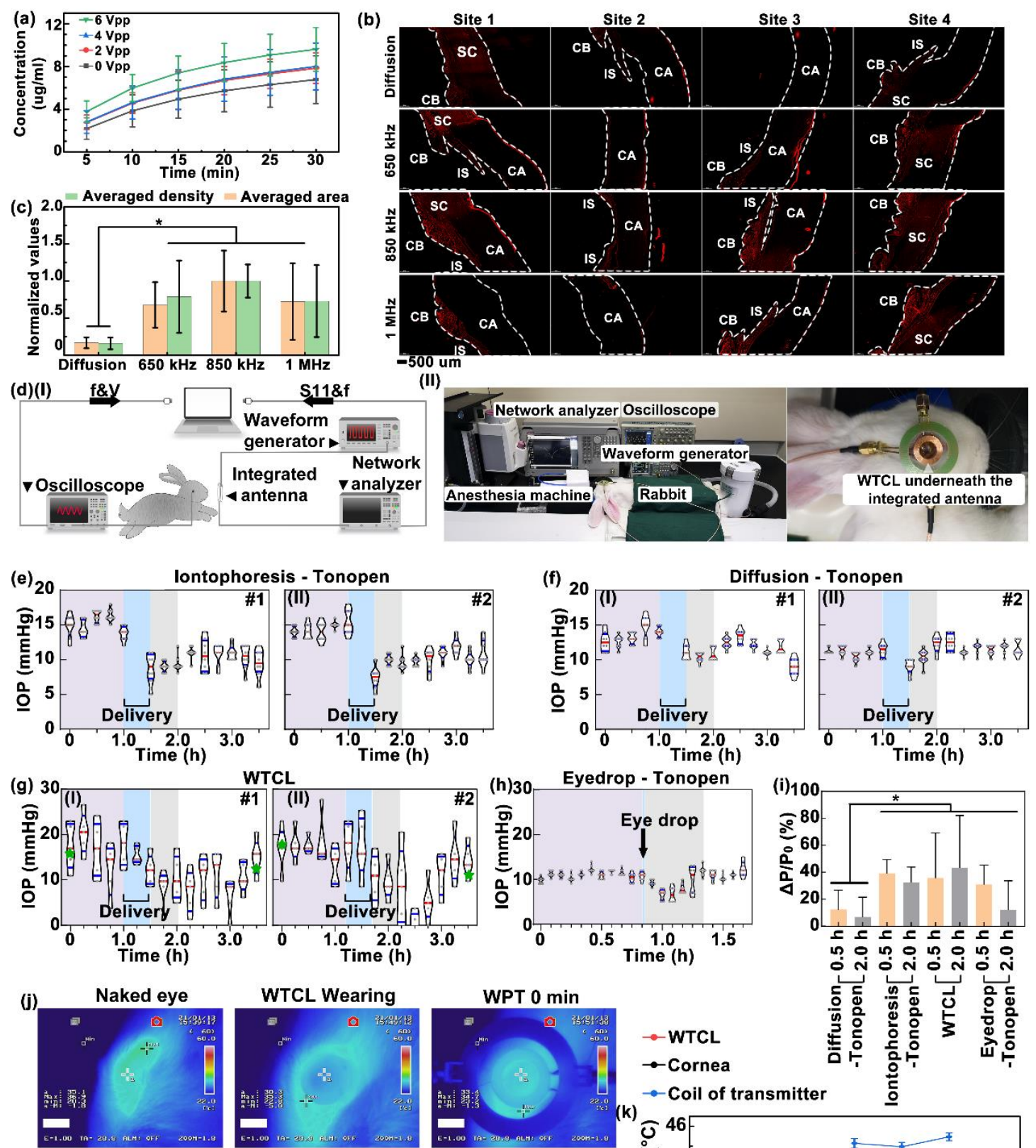

$\rightarrow$ WTCL

$\rightarrow$ Cornea

$\rightarrow$ Coil of transmitter

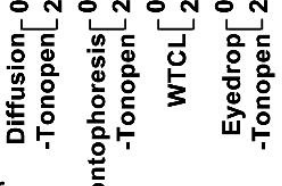

WPT $10 \mathrm{~min}$
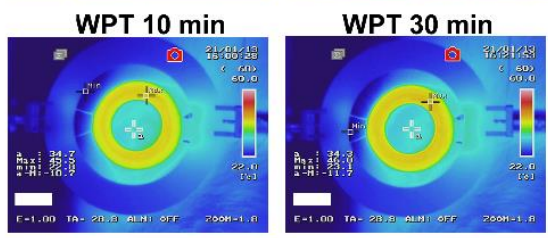

Naked eye

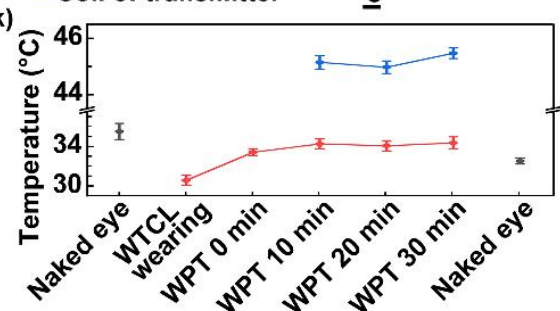

Figure 6. Sensing and therapeutic performance of the integrated WTCL. (a)

Quantitative analysis of in vitro rhodamine B released from WTCL at different alternating voltages for 30 min. $\mathrm{N}=6$ measurements. (b) Rhodamine $\mathrm{B}$ was utilized as the medicines analog in ex vivo experiments on porcine eyes, to examine the influence of iontophoresis on drug delivery across cornea. $\mathrm{CB}$, ciliary body; IS, iris; CA, Cornea. Scale bar is $500 \mu \mathrm{m}$. After delivery, fluorescence visualization in the anterior tissue was 
observed via microscope and (c) quantitatively analyzed. $\mathrm{N}=4$ sites per group. (d-I) Schematic and (II) experimental set-up of the in vivo WTCL experiments. The rabbits were anesthetized and worn with WTCL on their eyes, while the signals recording of WTCL and WPT operation were conducted by the integrated antenna. The rabbits' IOP were monitored with Tonopen, and brimonidine deliveries were performed via (e) Wirelessly powered iontophoresis or (f) passive free diffusion based on WTCL. (g) Simultaneous IOP sensing and drug delivery using a single WTCL device. The rabbit's IOP were wirelessly monitored with the WTCL, and brimonidine delivery via wireless iontophoresis on the same WTCL was conducted. The green asterisk indicated the IOP measurements via Tonopen for calibration or accuracy comparison. (h) Rabbit's eye was treated with eye drops of brimonidine, and the IOP was measured via Tonopen. $\mathrm{N}=2$ rabbits in (e)-(g) and $\mathrm{N}=1$ rabbit in (h). In (e)-(h), at each time point, 8 Topopen measurements were conducted, and the purple, blue, gray and white regions indicated the periods of prior to delivery, during delivery, $0.5 \mathrm{~h}$ after delivery and $2 \mathrm{~h}$ after delivery, respectively. (i) The IOP reductions effects after 0.5 and $2 \mathrm{~h}$ of drug delivery via iontophoresis, free diffusion and eye drops were summarized. Data were presented as mean \pm s.d. Significance was evaluated by one-way analysis of variance. $* \mathrm{P}<0.05$. (j) Monitoring of the thermal effects generated during WPT operation via infrared thermal camera, and (k) the temperate on cornea, WTCL, and transmitter coils during WPT process were analyzed. $\mathrm{N}=4$ measurements per group. Data were presented as mean \pm s.d. Significance was evaluated by one-way analysis of variance. ${ }^{*} \mathrm{P}<0.05$.

The in vitro release of brimonidine (Mw 292.1) from pHEMA hydrogel-coated electrode surface at different iontophoretic voltages (alternating voltages with 0-6 Vpp) was performed using a red fluorescent dye, rhodamine $\mathrm{B}$ ( $\mathrm{Mw} 479.0)$, as the medicines analog to facilitate quantifications via optical measurements. The results (Figure 6a) showed that the dye molecule was continuously released at a higher rate when higher voltages were applied, likely due to the fact that the electric field facilitated the diffusion of dye out of the hydrogel layer. Ex vivo experiments on porcine eyes were performed to examine the influence of iontophoresis on delivery across cornea, where 
rhodamine $\mathrm{B}$ was utilized as the medicines analog to facilitate visualization of distribution in tissue. The WTCL was worn on porcine eyes, and voltages with $6 \mathrm{Vpp}$ at $850 \mathrm{kHz}$ (the determined optimal WPT operation frequency) was applied to facilitate dye delivery via iontophoresis, and the anterior tissue was then fixed and sectioned for fluorescence visualization via microscope. Iontophoresis at other frequencies (650 and $1 \mathrm{MHz}$ ) or passive free diffusion were tested to optimize the iontophoresis conditions, and the fluorescence intensity and distribution area in the anterior tissues were analyzed. Red fluorescence was clearly observed in the tissues of ciliary body and anterior chamber angle for all the samples treated via iontophoresis (Figure 6b), while the group of free diffusion exhibited significantly (> 3-folds) lower fluorescence intensity and less (>3-folds) fluorescence distribution compared to the iontophoresis groups (Figure 6c). Of note, the ciliary body and anterior chamber angle have been proven to be the target sites for suppressing IOP by brimonidine through reducing aqueous humor production and increasing uveoscleral outflow. These results suggested the coupled iontophoresis could facilitate the delivery of drug analog molecules into anterior segment, and effectively work at the determined optimal WPT operation frequency of $850 \mathrm{kHz}$.

Next, in vivo experiments were conducted on rabbits, while the size of WTCL was proportionally scaled down to fit the rabbits' eyes. The WTCL was worn on the anesthetized rabbits' eyes, while the signals recording of WTCL and WPT operation were conducted by the integrated antenna (Figure 6d). The rabbits' IOP were monitored with either WTCL or commercial tonometry as a standard reference, and brimonidine delivery via wirelessly powered iontophoresis of WTCL was performed to reduce the IOP and compared to that via eyedrop. The initial IOP of rabbits exhibited slight fluctuation within the range of 10-15 $\mathrm{mmHg}$ as measured by Tonopen (Figure 6e), which rapidly $(<0.5 \mathrm{~h}$ ) dropped by $39.2 \pm 10.3 \%$ (Figure S6.5) after brimonidine delivery via wirelessly powered iontophoresis (at $6 \mathrm{Vpp}, 850 \mathrm{kHz}$, for $30 \mathrm{~min}$ ), and the IOP reduction remained above $20 \%$ for the prolonged period $(\sim 2 \mathrm{~h})$ after delivery (Figure S6.6). In contrast, brimonidine delivery via free diffusion (for $30 \mathrm{~min}$ ) from WTCL only slightly reduced IOP by $12.4 \pm 14.3 \%$ within $0.5 \mathrm{~h}$ after delivery (Figure $6 \mathrm{f}$ 
and Figure S6.5), and produced negligible effects $(6.85+14.7 \%)$ within $2 \mathrm{~h}$ (Figure S6.7). These results suggested that the slow diffusion of brimonidine from WTCL might form a basal delivery to stabilize the IOP, while iontophoresis was able to facilitate a bolus delivery to more effectively reduce IOP spikes. Simultaneous IOP sensing and drug delivery using a single WTCL device were next performed (Figure 6g). The rabbit's IOP were wirelessly monitored with the WTCL for the first hour, then in situ brimonidine delivery via wireless iontophoresis on the same WTCL was conducted to reduce IOP, which were still continuously monitored by the WTCL (Figure 6.1 and Figure S6.2). Considering the variations between in vitro and vivo sensing and the differences between rabbit' porcine' eyes, the rabbits' IOP were measured with Tonopen before experiments (at $\mathrm{t}=0 \mathrm{~h}$ ) to calibrate the WTCL's sensing results (Figure 6.3 and Figure S6.4). The last data points of IOP recorded by WTCL were compared to the reference IOP measured via Tonopen after experiments, and the results showed that the sensing error of WTCL was $<42 \%$. The IOP was observed to gradually drop by $32.5 \pm 35.9 \%$ (Figure S6.5) within 0.5 hour, and remained reduction of $43.2 \pm 38.8 \%$ (Figure S6.7) for the prolonged period. As control, eye drops of brimonidine (1 mg/ml, $50 \mathrm{uL}$ ) were instilled into rabbit's eye, and the IOP measured via Tonopen showed a reduction of $30.9 \pm 14.4 \%$ during a short period (<30 $\mathrm{min}$ ) (Figure S6.5), followed by rebounding rapidly to the initial IOP state. The IOP reductions effects via iontophoresis, free diffusion and eye drops were summarized in Figure 6e-i and Figure S6.5-6.7, and the results confirmed that the iontophoresis via WTCL rapidly reduce the IOP with pronounced and prolonged effects that was desirable for regulating glaucoma. At the end, since WPT operation at high frequency is likely to produce thermal effects that are harmful to animal eyes, the temperatures of rabbits' eye surface (cornea) and WTCL were monitored via infrared thermal camera during the process of WPT operation. The temperature of the and cornea was not increased, while the temperature of WTCL was observed to increase only by $<3{ }^{\circ} \mathrm{C}$, respectively, during WPT for $30 \mathrm{~min}$, suggesting negligible thermal effects produced by WTCL.

\section{Conclusion}


In this work, a soft, noninvasive and battery-free WTCL system for in situ IOP tracking and on-demand medicines administration was developed. The delicate design for structure, circuits layout of the device enabled highly integration on limited area and curved surface without causing vision blockage as well as potential irritations. The compact lens was exploited as a platform for deploying wireless bioelectronics and intimate contacting with human cornea, while the fabrication is compatible with highthroughput standard manufacturing process. The specialized design of frequency separation enabled individual operations of sensing and delivery modules without cross-coupling. Due to the unique cantilever configuration design of LCR circuit, the embedded wireless IOP sensor could ultra-sensitively detect IOP fluctuation, while the drug delivery modulus coupled with iontophoresis enabled highly efficient release of drug permeating across cornea. Systematic characterizations of IOP sensing, WPT, cross-coupling between individual sub-systems, iontophoretic medicines administration, and in vivo experiments all demonstrated the feasibility and promise of this WTCL platform for real-time monitoring and wireless controlled medical intervention. This smart system provides promising methodologies that could be expanded to other ophthalmic disease, which would positively promote the emerging of new generation of theranostic system for personalized health management.

\section{Methods}

Theoretical analysis of mutual inductance (M), power transfer efficiency $(\eta)$, and the skin effects. Mutual inductance $(M)$, a key factor in the technology of WPT, determines voltage in a secondary coil of receiver circuits. The critical parameter could be expressed as ${ }^{50}$ :

$$
M=k \sqrt{L_{1} L_{2}}
$$

where $L_{1}, L_{2}$ represent the inductance value of coil integrated in WPT transmitter and receiver circuit, respectively. $k$, denotes the magnetic coupling coefficient, means the link of magnetic flux between the WPT transmitter and receiver side ${ }^{43}$. The parameter is approximately equal to the following equation when the radiation distance is 
comparable to coils dimension ${ }^{43}$.

$$
k=\frac{1}{\left[1+2^{\frac{2}{3}}\left(\frac{d}{\sqrt{r_{1} r_{2}}}\right)^{2}\right]^{\frac{3}{2}}}
$$

where $d$ refers to distance between WPT transmitter and receiver circuit. Furthermore, $\mathrm{r}_{1}, \mathrm{r}_{2}$ denote radius of inductance coil of transmitter and receiver circuit.

According to these two equations mentioned above, the $M$ was inversely proportional to the radiation distance for these two coaxial coils of transmitter and receiver circuit. According to Kirchhoff's voltage law, the equation of the WPT system could be described as

$$
\left[\begin{array}{c}
U_{s} \\
0
\end{array}\right]=\left[\begin{array}{cc}
R_{P T}+j \omega L_{P T}-j \frac{1}{\omega C_{P T}} & -j \omega M \\
-j \omega M & R_{P R}+j \omega L_{P R}+\frac{Z_{L}}{1-j \omega C_{P R} Z_{L}}
\end{array}\right]\left[\begin{array}{c}
I_{P T} \\
I_{P R}
\end{array}\right]
$$

Where $U_{s}, R_{P T}, L_{P T}, C_{P T}, I_{P T}$ refer to the alternating voltage supplied for the transmitter, parasitic resistance, inductor, capacitor and alternating current in transmitter. Correspondingly, $R_{P R}, L_{P R}, C_{P R}, R_{L}$ denote the parasitic resistance, inductor, capacitor and electric load in receiver circuit. $I_{P R}$ represents the total alternating current in receiver circuit. $I_{L}$ is the alternating current flow through electric load.

To simplify the matrix, $\mathrm{Z}_{\mathrm{PT}}$ and $\mathrm{Z}_{\mathrm{PR}}$ were introduced as the impedance of transmitter and receiver circuit and expressed as

$$
\begin{gathered}
Z_{P T}=R_{P T}+j \omega L_{P T}-j \frac{1}{\omega C_{P T}}(2) \\
Z_{P R}=R_{P R}+j \omega L_{P R}+\frac{Z_{L}}{1-j \omega C_{P R} Z_{L}}
\end{gathered}
$$

Therefore, equation (1) could be transformed as

$$
\left[\begin{array}{c}
I_{P T} \\
I_{P R}
\end{array}\right]=\frac{U_{S}}{Z_{P T} Z_{P R}+\omega^{2} M^{2}}\left[\begin{array}{c}
Z_{P R} \\
j \omega M
\end{array}\right]
$$

And the current consumed by load illustrated as

$$
I_{L}=\frac{j \omega M U_{S}}{\left(1-j \omega C_{P R} Z_{L}\right)\left(Z_{P T} Z_{P R}+\omega^{2} M^{2}\right)}
$$

The power transfer efficiency $\eta$ was regarded to be the ratio of the real power dissipated in the load impedance $P_{\text {out }}$ to the power supplied from the source side $P_{\text {in }}$, 


$$
\eta=\frac{P_{o u t}}{P_{\text {in }}}=\frac{\omega^{2} M^{2} Z_{L}}{Z_{P R}\left(1+\omega^{2}{C_{P R}}^{2} Z_{L}^{2}\right)\left(Z_{P T} Z_{P R}+\omega^{2} M^{2}\right)}
$$

As regards high frequency circuit, alternating high-frequency currents tend to distributed toward the surface of conductor. This phenomenon, known as skin effect, will increase the resistance of the conductor and reduce the effective electric power exerted on load. The effective cross-section of the conductor for alternating currents was defined as skin depth that could be expressed by the following equation:

$$
\delta=\left(\pi f \mu_{r} \mu_{0} \sigma\right)^{-\frac{1}{2}}
$$

where $\delta, f, \mu_{r}, \mu_{0}$, and $\sigma$ represent skin depth in meters, frequency of the alternating current in $\mathrm{Hz}$, relative magnetic permeability of the conductive matter, permeability of free space $\left(4 \pi \times 10^{-7} \mathrm{H} / \mathrm{m}\right)$, and conductivity of conductor. Detailed parameters (relative magnetic permeability and conductivity of the conductive matter) were listed in Table S4.

Fabrication of IOP monitoring circuits. The sensing and delivery were designed, and the fabricating process including Copper $(\mathrm{Cu})$ film deposition, photolithography, etching, and laser cutting were performed by Shenzhen Gaoyue Electronics Co. Ltd, China. $\mathrm{Cu}$ film deposition process (including sputtering and electrical plating) was performed to establish electric film on the surface of PI substrate. After that, $\mathrm{Cu}$ film was patterned by photolithography and development process. Extra $\mathrm{Cu}$ film was etched by to form $\mathrm{Cu}$ electrodes, which were then covered with nickel (Ni) and gold (Au) to improve biocompatibility for the flexible circuits. Subsequently, ultraviolet beam excited by high energy YAG laser was utilized to cut the PI substrate to form the snowflake-shaped layout for the flexible IOP sensing circuits.

Fabrication of upper lens. Each capacitive sensing plate (totally 6 plates) of the flexible IOP sensing circuit was aligned with reference plate and folded manually. Then the folded IOP sensing circuit was positioned into the metal mold for contact lens. Polydimethylsiloxane (PDMS, Sylgard 184, Dow Corning) and curing agent were 
prepared according to the ration of 10:1 and then stirred sufficiently. The transparent PDMS solution was placed in vacuum with pressure of $10 \mathrm{~Pa}$ for $30 \mathrm{~min}$ to remove bubbles, and injected into the metal mold. After vacuum treatment (10 $\mathrm{Pa}, 30 \mathrm{~min}$ ), the upper mold and bottom mold were assembled and placed into oven $\left(80^{\circ} \mathrm{C}, 1.5 \mathrm{~h}\right)$. Afterwards, the contact lens embedded with IOP monitoring circuit was disassembled from mold carefully. Finally, sensing plates were detached from the upper contact lens manually. While reference plates and 5 coils of inductance were kept inside the upper lens. The dangling sensing plates aligned with reference plates served as cantilever configuration.

Fabrication, soldering of drug delivery circuits. The fabricating process contained film deposition, electrical plating, chemical plating, photolithography, etching, drilling, and laser cutting (by Shenzhen Gaoyue Electronics Co. Ltd, China). Cu film deposition process (including chemical and electrical plating) was performed to establish electric film on the surface of PI substrate. After that, $\mathrm{Cu}$ film was patterned by photolithography and development process and etched to form $\mathrm{Cu}$ electrodes, and the surface except the iontophoretic electrodes were insulated by a thin layer of PI. Ultraviolet laser with high energy was adopted to fabricate through-hole on PI substrate. Chemical and electrical plating were conducted to deposit $\mathrm{Cu}$ film on the surface of PI substrate and through-hole, which enables electric connections between electrodes on the top and bottom layers. Sequentially, photolithography, development and wet etching process were performed to form patterned $\mathrm{Cu}$ electrodes that were then covered with $\mathrm{Ni}$ and $\mathrm{Au}$ layer to improve biocompatibility for the flexible circuits. High energy laser was utilized to cut the PI substrate to define the flower-shaped layout for drug delivery circuits. Subsequently, ceramic chip capacitors ( $1 \mathrm{~mm}$ length, $0.5 \mathrm{~mm}$ width, $0.5 \mathrm{~mm}$ thickness) were attached to their respective sites on flexible circuits using low temperature solder by electrical soldering iron. 
Fabrication of bottom lens. Drug delivery circuit was positioned in metal mold for contact lens. PDMS solution was placed in vacuum with pressure of $10 \mathrm{~Pa}$ for $30 \mathrm{~min}$ to remove bubbles, and injected into the mold. After vacuum treatment (10 $\mathrm{Pa}, 30 \mathrm{~min})$, the top and bottom molds were assembled and placed into oven $\left(80{ }^{\circ} \mathrm{C}, 1.5 \mathrm{~h}\right)$. Afterwards, the PDMS contact lens integrated with drug delivery circuit was disassembled from mold carefully. Finally, extra PDMS film covered on iontophoretic electrodes (including delivery and counter electrodes) was removed manually.

WTCL integration and drug loading. The upper lens integrated with IOP monitoring circuit and bottom lens embedded with drug delivery circuit were assembled by liquid PDMS glue in oven $\left(60^{\circ} \mathrm{C}, 3 \mathrm{~h}\right)$. The materials for preparing pHEMA hydrogel preparation included HEMA monomer (1.45 ml), EGDMA $(5 \mu \mathrm{l})$ as crosslinker, DI water $(0.5 \mathrm{ml})$, and brimonidine tartrate $(10 \mathrm{mg})$. Darocur $(6 \mathrm{mg})$, a photoinitiator was mixed into monomer mixture and sonicated. The mixture solution of pHEMA hydrogel $(10 \mu \mathrm{l})$ loaded with brimonidine tartrate $(5 \mathrm{mg} / \mathrm{ml})$ was drop-casted onto the drug delivery electrode. The solution was irradiated with UVB light $(365 \mathrm{~nm})$ for 20 min for the hydrogel polymerization, and kept at room temperature overnight.

Characterization of circuits. Microscopic images of IOP sensing and drug delivery circuits were captured by inverted fluorescence microscope (MF52-N, Guangzhou Micro-shot Technology Co., Ltd, China)

In-vitro performance of wireless IOP monitoring. The porcine eyes were placed in the lab with room temperature $\left(27 \pm 3{ }^{\circ} \mathrm{C}\right)$ and humidity $(50 \pm 10 \%)$ to avoid the shape changes of eyeball induced by water loss. Before the deployment of wearable smart contact lens, physiological saline solution $(200 \mu \mathrm{l})$ was dript on the surface of cornea to build a water film. The layer could be used to simulate tear film to avoid bubble between cornea and contact lens. IOPs ranging from 5 to $50 \mathrm{mmHg}$ were achieved inside the eye by injecting saline solution into the anterior chamber via a disposable 
intravenous infusion needles $(0.45 \times 13.5 \mathrm{~mm})$ controlled by syringe pump (PHD ULTRA, Harvard Apparatus, Inc., U.S.A.) During the experiments, a pressure gauge (GM511, Shenzhen Jumaoyuan Science And Technology Co., Ltd, China) was connected to the anterior chamber by a disposable intravenous infusion needle to independently track the value of IOP. The resonance frequency of the IOP monitoring module was record wirelessly by IOP reading coil (diameter: $17 \mathrm{~mm}$, turns: 1 ) of the integrated antenna connected to a network analyzer (E5063A, Agilent Technologies Inc., Santa Clara, CA, USA).

Dynamical responsion of wireless IOP monitoring. Physiological saline solution was injected into the anterior chamber of porcine eye to elevate the IOP from $4.5 \mathrm{mmHg}$ to $30 \mathrm{mmHg}$. Then, the pressure decreases down to $13 \mathrm{mmHg}$ with the continuous leaking of solution from eyeball. Sequentially, saline was filled into in vitro eyeball again to raise the pressure again. During this process, resonant frequency changes of the IOP monitoring module in WTCL were recorded wirelessly by network analyzer, and the pressure data in anterior chamber was validated using a commercial pressure gauge via a disposable intravenous infusion needle.

WPT performance characterization of the WTCL system. During the measurements, integrated antenna was immobilized above WTCL deployed on porcine eye. The in vitro organ that was posited on cystosepiment board held by a multi-axis stages to adjust the distance between WTCL and integrated antenna. During the collection of scattering parameter, two ports of vector network analyzer (E5063A, Keysight Technologies, USA) was connected to the WPT transmitter of the integrated antenna. Four different receivers (Rec\#2, Rec\#5, Rec\#9, and Rec\#17 integrated in four WTCLs) were connected to network analyzer successively to conduct data collections. During the measurements of wireless voltage transfer performance, waveform generator (DG1022, Beijing RIGOL Technology Co., Ltd., China) was adopted as power source for operating the WPT transmitter of the integrated antenna. Oscilloscope (TDS2014C, 
Tektronix, USA) was connected to WPT receiver circuit of WTCL for recording the voltage signal collected wirelessly.

\section{Characterization of disturbance generated by the radiation of WPT transmitter} to IOP sensing module. Saline solution were injected into the anterior chamber of in vitro porcine eyes via a infusion needles $(0.45 \times 13.5 \mathrm{~mm})$ controlled by syringe pump to achieve IOPs ranging from 5 to $50 \mathrm{mmHg}$. Network analyzer was connected to the IOP reading coil of the integrated antenna to monitor the physiological pressure transduced by WTCL. The distance between integrated antenna and WTCL was set as $6 \mathrm{~mm}$. A commercial pressure gauge was exploited to validate the shifts of pressure inside of the anterior chamber through a infusion needles $(0.45 \times 13.5 \mathrm{~mm})$. Moreover, waveform generator was connected to WPT transmitter of the integrated antenna. During the process of IOP sensing, the power of waveform generator to support WPT transmitter was turned on and off to observe and record the S11 response of the IOP monitoring module.

\section{Characterization of WPT performance of Rec\#17 integrated in WTCL under the radiation of} IOP reading coil. During the measurements, integrated antenna was immobilized above WTCL deployed on porcine eye. The distance between integrated antenna and WTCL was set as $6 \mathrm{~mm}$. The IOP reading coil and Rec\#17 integrated in WTCL was connected to two ports of network analyzer for the recording of the S21 parameters. As a control group, IOP reading coil was replaced by WPT transmitter that was coupled with Rec\#17 to record S21 parameters. Similarly, The IOP reading coil of integrated antenna was connected to waveform generator. While, the Rec\#17 circuit integrated in WTCL was connected to oscilloscope for the collecting of voltage signal. Correspondingly, WPT transmitter was adopted to replace the IOP sensing coil. Moreover, integrated antenna (including IOP reading coil and WPT transmitter) was disconnected from waveform generator, while Rec\#17 was still connected with oscilloscope. The collected data serve as blank group. 


\section{Theoretical simulations of iontophoretic medicines administration via COMSOL}

Multiphysics 5.5. The theoretical simulations of iontophoretic medicines administration were performed with COMSOL Multiphysics software using the AC/DC module and Chemical Species Transport module. To visualize the effect of active agents administration and spatial distributions of electric potential, the process of cargo delivery were simulated with a 3D model, where the components and geometric layouts mimicked the actual experimental setup. Anterior segment of the eye was modeled as aqueous humor, cornea including epithelial cell, stroma, and endothelial cell layer. Counter electrode and pHEMA hydrogel that served as reservoir to load bio-active compounds were attached on cornea. Furthermore, the back surface of hydrogel was set as a drug delivery electrode to generate electric potential. Correspondingly, the counter electrode was labeled with ground in electric field. Under the action of constant electrical voltages, the working electrode combined with counter electrode form electric field through the tissue of cornea. For drug delivery, the drug concentration was set as $\mathrm{Cg} 0$ in hydrogel, and gradually diffused into aqueous humor through corneal barriers facilitated by electric field. After that, the average compounds concentration in the anterior chamber (aqueous humor) was calculated to evaluate the drug delivery efficiency. The bio-active molecules concentration was then normalized by comparing to the initial cargo's concentration loaded in hydrogel. Critical factors in this simulation work involves: 1) the drug diffusivities and the electrical conductivities in the pHEMA hydrogel, corneal layers, and aqueous humor; 2) Electrical charge of drugs. Detailed physic setting of cargo administrations, related parameters were demonstrated in table $\mathrm{S} 5$ in this supporting information file.

AC/DC module was exploited to simulate the steady electric filed distribution, which was performed by electric currents interface, following the theoretical equation:

$$
\begin{gathered}
\nabla \cdot J=0 \\
J=\sigma E \\
E=-\nabla V
\end{gathered}
$$


Where $\mathrm{V}$ denotes potential, E refers to the intensity of electric field, J represents current density, $\sigma$ is the material conductivity, $\nabla$ refers to Hamiltonian. These equations mentioned above contributed to a Laplace equation that could be adopted to calculate electric potential and electric field in this model:

$$
\nabla(\sigma \cdot \nabla V)=0
$$

On the top boundary of drug delivery electrode, a boundary voltage terminal was used to simulate the constant voltage source:

$$
V=V_{0}
$$

Where $V_{0}$ refers to constant voltage.

Then, dynamic iontophoresis process was simulated by Chemical Species Transport module according to the electric filed distribution. The theoretical equation could be expressed as:

$$
\begin{gathered}
\frac{\partial c}{\partial t}+\nabla \cdot J_{t d s}=0 \\
J_{t d s}=-D_{e} \nabla_{C}-z u_{m e} F c \nabla V
\end{gathered}
$$

Where $J_{t d s}$ refers to diffusion flux vector, c denotes the concentration, $\mathrm{z}$ represents the charge number, $\mathrm{F}$ refers to Faraday constant, $\mathrm{V}$ is electric potential, $D_{e}$ corresponding to the effective diffusion coefficient, $u_{m e}$ denotes the effective mobility. Therefore, the relationship of $D_{e}$ and $u_{m e}$ can be demonstrated by Nernst-Einstein equation:

$$
u_{m e}=\frac{D e}{R T}
$$

Where $\mathrm{R}$ represents Moore gas constant, $\mathrm{T}$ is temperature.

Quantitative analysis of rhodamine B released from WTCL. In this work, rhodamine B was exploited as the medicines analog to visualize the distribution of medicines in bio-tissue. Rhodamine B in PBS solution with the concentration of 0.5 to $30 \mathrm{ug} / \mathrm{ml}$ was prepared, and the standard curve of absorption value and solution's concentration was established. Then HEMA monomer (1.45 ml), EGDMA (5 $\mu \mathrm{l})$ as crosslinker, DI water $(0.5 \mathrm{ml})$, photoinitiator Darocur $(9 \mathrm{mg})$ were mixed. The mixture solution of pHEMA hydrogel $(10 \mu \mathrm{l})$ loaded with rhodamine B $(0.4 \mathrm{mg} / \mathrm{ml})$ was drop- 
casted onto the drug delivery electrode of WTCL. After that, the solution coated on WTCL was irradiated with UVB light $(365 \mathrm{~nm})$ for $20 \mathrm{~min}$ for the hydrogel polymerization, and kept at room temperature overnight. The WTCL connected with oscilloscope, and powered by the WPT transmitter of the integrated antenna underneath the WTCL (distance: $6 \mathrm{~mm}$ ). $200 \mu \mathrm{PBS}$ solution was placed on the WTCL to allow dye diffusion, and $100 \mu \mathrm{l}$ solution was withdrawn every 5 minutes for analysis of the diffusion rate. Accordingly, fresh PBS with equal volume was re-supplemented into WTCL. These absorbance values of solutions collected at each time point was measured by microplate reader. According to these absorbance values, the accumulated concentration of released rhodamine B could be calculated according to the equation mentioned above.

Ex vivo delivery of Rhodamine B in to porcine eyes by WTCL. The WTCL was worn on in vitro porcine eye, and the integrated antenna was deployed on top of WTCL with the distance of $6 \mathrm{~mm}$. Waveform generator was connected to the WPT transmitter of integrated antenna as power source. Oscilloscope was connected to the WPT Rec\#17circuit in WTCL to monitor the voltage adopted for iontophoresis and ensure that the received alternating voltage stabilized around $6 \mathrm{Vpp}$ with the frequency of 650 $\mathrm{kHz}, 850 \mathrm{kHz}$, and $1 \mathrm{MHz}$, respectively. Moreover, drug delivery in the manner of free diffusion was performed as a control group. A through-hole (diameter: $4 \mathrm{~mm}$ ) was created in the central area of WTCL to allow dropping of PBS solution to the eye surface for maintaining humidity. During experiments, PBS was instilled into the central hole of WTCL with the speed of $30 \mu \mathrm{L}$ every $30 \mathrm{~s}$ to form a thin solution film on the corneal surface. The liquid film has been regarded as simulant of tears to ensure reliable connection between drug delivery and cornea electrically, and also prevent drying of ocular surface tissue ${ }^{39}$. After the completion of examinations, the corneal surface of porcine eye was irrigated by PBS. Then extra tissues (muscle, fat) outside of eyeball were removed by dissecting scissors. The whole eyeball was fixed in a paraformaldehyde solution (Fixative Solution, 4\% formaldehyde, methanol-free, 
Biosharp Co., Ltd, China), sectioned, stained by Wuhan Servicebio Technology Co., Ltd. Sequentially, fluorescent microscopic images including bio-tissue of ciliary body, iris, cornea (site 1-4) in each experiment condition (free diffusion, and $6 \mathrm{Vpp}$ with the frequency of $650 \mathrm{kHz}, 850 \mathrm{kHz}, 1 \mathrm{MHz}$ ) were taken and processed by Image J program to quantify the fluorescence intensity and distribution area in the anterior tissues. The mean distribution area and integrated density of rhodamine B in the sample treated by iontophoretic drug administration with $20 \mathrm{Vpp}$ at $850 \mathrm{kHz}$ were set to be base reference of 1 for normalization. Correspondingly, normalized values of distribution area and integrated density of rhodamine B in the sample treated by other drug delivery conditions could be quantified.

Animal experiments. Male New Zealand white rabbits weighing about $3 \mathrm{~kg}$ (Animal Center, Sun Yat-sen University, Guangzhou, China), adopted for in vivo experiments, were maintained in climate-controlled independent room with $12 \mathrm{~h} / 12 \mathrm{~h}$ light/dark cycle separately. All in vivo experiments in this study were reviewed, permitted, and supervised by the Institutional Animal Care and Use Committee of the Sun Yat-sen University. For all in vivo experiment process, the rabbits were deeply anesthetized with pentobarbital sodium solution $(0.8 \mathrm{ml} / \mathrm{kg}$ body weight). To minimize side effects, the administration of anesthetic solution was separately into three times through ear venous and twice intramuscular injection every ten minutes successively. Moreover, Isoflurane and oxygen were supplied through gas anaesthesia machine for rabbit to obtain prolonged anesthesia effects. Propivacaine hydrochloride eye drops (S. A. ALCON-COUVREUR N.V. Belgium) were dropped onto the rabbit cornea surface for topical anesthesia to avoid ocular movement including blink, facilitate WTCL wearing and IOP measurement by commercial ophthalmotonometer. 
In vivo experiments of WTCL performance. Pentobarbital sodium (Nembutal, Ovation Pharmaceuticals Inc. Deerfield, USA) solution in saline (0.3 wt\%) was prepared. New Zealand white rabbit $(3 \mathrm{~kg}$ ) was initially anesthetized with an appropriate dose of pentobarbital sodium solution $(0.8 \mathrm{ml} / \mathrm{kg}$ body weight $)$, and continuously anesthetized with Isoflurane anesthesia machine. To avoid unexpected situations, the administration of anesthetic solution was divided into three times through ear venous and twice intramuscular injection every ten minutes successively. After anesthesia, the rabbits were covered with blanket to maintain body temperature. Propivacaine hydrochloride eye drops were dropped onto the rabbit cornea surface for local anesthesia to further avoid of ocular movement including blink. Commercial applanation tonometer (Tono-Pen Avia; Reichert, Inc., Depew, NY) was applied to acquire IOP measurement as reference. WTCL was worn on rabbit's eye, and oscilloscope was connected to the WTCL to monitor the Vpp between delivery and counter electrode during WPT process. Integrated antenna connected to network analyzer and waveform generator was posited above WTCL with the distance of $6 \mathrm{~mm}$. Sequentially, measurements of return loss by IOP reading coil was collected by network analyzer to wirelessly detect IOP. After one hour, square voltage with $20 \mathrm{Vpp}$ at 850 $\mathrm{kHz}$ produced from waveform generator was exerted on WPT transmitter to trigger iontophoretic delivery wirelessly. Meanwhile, wireless IOP monitoring was continuously performed until the end of experiments.

Thermal characterization. New Zealand white rabbit $(3 \mathrm{~kg})$ was anesthetized with an appropriate dose of pentobarbital sodium solution $(0.8 \mathrm{ml} / \mathrm{kg}$ body weight) through ear venous injections. After general anesthesia, the rabbits were covered with blanket to maintain body temperature. Then anesthesia machine was further adopted to supply isoflurane and oxygen via facemask for the rabbit, which could obtain prolonged anesthesia effects. WTCL was worn on rabbit's eye, and integrated antenna connected to waveform generator was posited above WTCL with the distance of $6 \mathrm{~mm}$. Square voltage with $20 \mathrm{Vpp}$ at $850 \mathrm{kHz}$ produced from waveform generator was exerted on 
WPT transmitter. Infrared camera (T650sc, FLIR Systems, Wilsonille, OR, USA) was exploited to monitor thermal changes of ocular surface tissue, WTCL, and integrated antenna during the experimental process.

\section{References}

1 Lee, H., Song, C., Hong, Y. S., Kim, M. S., Cho, H. R., Kang, T., Shin, K., Choi, S. H., Hyeon, T. \& Kim, D.-H. Wearable/disposable sweat-based glucose monitoring device with multistage transdermal drug delivery module. Sci. Adv. 3, e1601314 (2017).

2 Keum, D. H., Kim, S.-K., Koo, J., Lee, G.-H., Jeon, C., Mok, J. W., Mun, B. H., Lee, K. J., Kamrani, E., Joo, C.-K., Shin, S., Sim, J.-Y., Myung, D., Yun, S. H., Bao, Z. \& Hahn, S. K. Wireless smart contact lens for diabetic diagnosis and therapy. Sci. Adv. 6, eaba3252 (2020).

3 Lee, H., Choi, T. K., Lee, Y. B., Cho, H. R., Ghaffari, R., Wang, L., Choi, H. J., Chung, T. D., Lu, N., Hyeon, T., Choi, S. H. \& Kim, D.-H. A graphene-based electrochemical device with thermoresponsive microneedles for diabetes monitoring and therapy. Nat. Nanotechnol. 11, 566572 (2016).

4 Son, D., Lee, J., Qiao, S., Ghaffari, R., Kim, J., Lee, J. E., Song, C., Kim, S. J., Lee, D. J., Jun, S. W., Yang, S., Park, M., Shin, J., Do, K., Lee, M., Kang, K., Hwang, C. S., Lu, N., Hyeon, T. \& Kim, D.-H. Multifunctional wearable devices for diagnosis and therapy of movement disorders. Nat. Nanotechnol. 9, 397-404 (2014).

5 Han, M., Chen, L., Aras, K., Liang, C., Chen, X., Zhao, H., Li, K., Faye, N. R., Sun, B., Kim, J.-H., Bai, W., Yang, Q., Ma, Y., Lu, W., Song, E., Baek, J. M., Lee, Y., Liu, C., Model, J. B., Yang, G., Ghaffari, R., Huang, Y., Efimov, I. R. \& Rogers, J. A. Catheter-integrated soft multilayer electronic arrays for multiplexed sensing and actuation during cardiac surgery. Nat. Biomed. Eng 4, 997-1009 (2020).

6 Mickle, A. D., Won, S. M., Noh, K. N., Yoon, J., Meacham, K. W., Xue, Y., McIlvried, L. A., Copits, B. A., Samineni, V. K., Crawford, K. E., Kim, D. H., Srivastava, P., Kim, B. H., Min, S., Shiuan, Y., Yun, Y., Payne, M. A., Zhang, J., Jang, H., Li, Y., Lai, H. H., Huang, Y., Park, S.-I., Gereau, R. W. \& Rogers, J. A. A wireless closed-loop system for optogenetic peripheral neuromodulation. Nature 565, 361-365 (2019).

7 Weinreb, R. N., Leung, C. K. S., Crowston, J. G., Medeiros, F. A., Friedman, D. S., Wiggs, J. L. \& Martin, K. R. Primary open-angle glaucoma. Nat. Rev. Dis. Primers 2, 16067 (2016).

8 Jonas, J. B., Aung, T., Bourne, R. R., Bron, A. M., Ritch, R. \& Panda-Jonas, S. Glaucoma. Lancet 390, 2183-2193 (2017).

9 Wang, N., Chintala, S. K., Fini, M. E. \& Schuman, J. S. Activation of a tissue-specific stress response in the aqueous outflow pathway of the eye defines the glaucoma disease phenotype. Nat. Med. 7, 304-309 (2001).

Hughes, E., Spry, P. \& Diamond, J. 24-hour monitoring of intraocular pressure in glaucoma management: A retrospective review. J. Glaucoma 12, 232-236 (2003).

Taylor, S. A., Galbraith, S. M. \& Mills, R. P. Causes of non-compliance with drug regimens in glaucoma patients: A qualitative study. J. Ocul. Pharmacol. Ther. 18, 401-409 (2002).

Freedman, S. F., Beck, A. D., Nizam, A., Vanderveen, D. K., Plager, D. A., Morrison, D. G., Drews-Botsch, C. D., Lambert, S. R. \& Infant Aphakia Treatment Study, G. Glaucoma-Related 
Adverse Events at 10 Years in the Infant Aphakia Treatment Study A Secondary Analysis of a Randomized Clinical Trial. JAMA Ophthalmol. 139, 165-173 (2021). Grehn;, F. \& Stamper;, R. Glaucoma : [progress III]. (Springer, 2006).

Agaoglu, S., Diep, P., Martini, M., Samudhyatha, K. T., Baday, M. \& Araci, I. E. Ultra-sensitive microfluidic wearable strain sensor for intraocular pressure monitoring. Lab Chip 18, 34713483 (2018).

Morrison;, J. C. \& Pollack; I. P. Glaucoma: Science and Practice. (Thieme, 2003).

Yang, H., Tyagi, P., Kadam, R. S., Holden, C. A. \& Kompella, U. B. Hybrid Dendrimer Hydrogel/PLGA Nanoparticle Platform Sustains Drug Delivery for One Week and Antiglaucoma Effects for Four Days Following One-Time Topical Administration. Acs Nano 6, 7595-7606 (2012).

Novack, G. D. Ophthalmic Drug Delivery: Development and Regulatory Considerations. Clin. Pharmacol. Ther. 85, 539-543 (2009).

Richa, S. \& Yazbek, J. C. Ocular Adverse Effects of Common Psychotropic Agents A Review. CNS Drugs 24, 501-526 (2010).

Farandos, N. M., Yetisen, A. K., Monteiro, M. J., Lowe, C. R. \& Yun, S. H. Contact Lens Sensors in Ocular Diagnostics. Adv. Healthc. Mater. 4, 792-810 (2015).

Tseng, R. C., Chen, C. C., Hsu, S. M. \& Chuang, H. S. Contact-Lens Biosensors. Sensors 18, 2651 (2018).

Kim, J., Kim, J., Ku, M., Cha, E., Ju, S., Park, W. Y., Kim, K. H., Kim, D. W., Berggren, P.-O. \& Park, J.-U. Intraocular Pressure Monitoring Following Islet Transplantation to the Anterior Chamber of the Eye. Nano Lett. 20, 1517-1525 (2020).

Maeng, B., Chang, H.-k. \& Park, J. Photonic crystal-based smart contact lens for continuous intraocular pressure monitoring. Lab Chip 20, 1740-1750 (2020).

Park, J., Kim, J., Kim, S.-Y., Cheong, W. H., Jang, J., Park, Y.-G., Na, K., Kim, Y.-T., Heo, J. H., Lee, C. Y., Lee, J. H., Bien, F. \& Park, J.-U. Soft, smart contact lenses with integrations of wireless circuits, glucose sensors, and displays. Sci. Adv. 4, eaap9841 (2018).

Elsherif, M., Hassan, M. U., Yetisen, A. K. \& Butt, H. Glucose Sensing with Phenylboronic Acid Functionalized Hydrogel-Based Optical Diffusers. Acs Nano 12, 2283-2291 (2018).

Kim, J., Kim, M., Lee, M.-S., Kim, K., Ji, S., Kim, Y.-T., Park, J., Na, K., Bae, K.-H., Kim, H. K., Bien, F., Lee, C. Y. \& Park, J.-U. Wearable smart sensor systems integrated on soft contact lenses for wireless ocular diagnostics. Nat. Commun. 8, 14997 (2017).

Kim, J., Park, J., Park, Y.-G., Cha, E., Ku, M., An, H. S., Lee, K.-P., Huh, M.-I., Kim, J., Kim, T.-S., Kim, D. W., Kim, H. K. \& Park, J.-U. A soft and transparent contact lens for the wireless quantitative monitoring of intraocular pressure. Nat. Biomed. Eng (2021).

Kim, H.-J., Zhang, K., Moore, L. \& Ho, D. Diamond Nanogel-Embedded Contact Lenses Mediate Lysozyme-Dependent Therapeutic Release. Acs Nano 8, 2998-3005 (2014).

Jung, H. J. \& Chauhan, A. Temperature sensitive contact lenses for triggered ophthalmic drug delivery. Biomaterials 33, 2289-2300 (2012).

Guzman, G., Es-haghi, S. S., Nugay, T. \& Cakmak, M. Zero-Order Antibiotic Release from Multilayer Contact Lenses: Nonuniform Drug and Diffusivity Distributions Produce ConstantRate Drug Delivery. Adv. Healthc. Mater. 6, 1600775 (2017).

30 Janagam, D. R., Wu, L. \& Lowe, T. L. Nanoparticles for drug delivery to the anterior segment of the eye. Adv. Drug Deliv. Rev. 122, 31-64 (2017). 

Antibacterial Conductive Hydrogels for Epidermal Sensors and Diabetic Foot Wound Dressings. Adv. Funct. Mater. 29, 1901474 (2019).

Sim, K., Chen, S., Li, Z., Rao, Z., Liu, J., Lu, Y., Jang, S., Ershad, F., Chen, J., Xiao, J. \& Yu, C. Three-dimensional curvy electronics created using conformal additive stamp printing. Nature Electronics 2, 471-479 (2019).

Guo, S., Wu, K., Li, C., Wang, H., Sun, Z., Xi, D., Zhang, S., Ding, W., Zaghloul, M. E., Wang, C., Castro, F. A., Yang, D. \& Zhao, Y. Integrated contact lens sensor system based on multifunctional ultrathin MoS2 transistors. Matter 4, 969-985 (2021).

Park, J., Ahn, D. B., Kim, J., Cha, E., Bae, B.-S., Lee, S.-Y. \& Park, J.-U. Printing of wirelessly rechargeable solid-state supercapacitors for soft, smart contact lenses with continuous operations. Sci. Adv. 5, eaay0764 (2019).

Yuan, M., Das, R., Ghannam, R., Wang, Y., Reboud, J., Fromme, R., Moradi, F. \& Heidari, H. Electronic Contact Lens: A Platform for Wireless Health Monitoring Applications. Adv. Intell. Syst. 2, 1900190 (2020).

Boutry, C. M., Beker, L., Kaizawa, Y., Vassos, C., Tran, H., Hinckley, A. C., Pfattner, R., Niu, S., Li, J., Claverie, J., Wang, Z., Chang, J., Fox, P. M. \& Bao, Z. Biodegradable and flexible arterial-pulse sensor for the wireless monitoring of blood flow. Nat. Biomed. Eng 3, 47-57 (2019).

Chen, G.-Z., Chan, I.-S. \& Lam, D. C. C. Capacitive contact lens sensor for continuous noninvasive intraocular pressure monitoring. Sens. Actuator A-Phys. 203, 112-118 (2013).

Qi, D., Liu, Z., Liu, Y., Jiang, Y., Leow, W. R., Pal, M., Pan, S., Yang, H., Wang, Y., Zhang, X., Yu, J., Li, B., Yu, Z., Wang, W. \& Chen, X. Highly Stretchable, Compliant, Polymeric Microelectrode Arrays for In Vivo Electrophysiological Interfacing. Adv. Mater. 29 (2017). Christopher, K. \& Chauhan, A. Delivery of ionic molecules to anterior chamber by iontophoretic contact lenses. Eur. J. Pharm. Biopharm. 140, 40-49 (2019).

40 Kurs, A., Karalis, A., Moffatt, R., Joannopoulos, J. D., Fisher, P. \& Soljacic, M. Wireless power transfer via strongly coupled magnetic resonances. Science 317, 83-86 (2007).

Noh, K. N., Park, S. I., Qazi, R., Zou, Z., Mickle, A. D., Grajales-Reyes, J. G., Jang, K.-I., Gereau, R. W., Xiao, J., Rogers, J. A. \& Jeong, J.-W. Miniaturized, Battery-Free Optofluidic Systems with Potential for Wireless Pharmacology and Optogenetics. Small 14, 1702479 (2018). Bansal, R. Antenna theory; analysis and design. Proceedings of the IEEE 72, 989-990 (1984). Imura;, T. Wireless Power Transfer Using Magnetic and Electric Resonance Coupling Techniques. (Springer, 2020).

44 Sempionatto, J. R., Lin, M., Yin, L., de la Paz, E., Pei, K., Sonsa-ard, T., Silva, A. N. d. L., Khorshed, A. A., Zhang, F., Tostado, N., Xu, S. \& Wang, J. An epidermal patch for the simultaneous monitoring of haemodynamic and metabolic biomarkers. Nat. Biomed. Eng (2021).

Gaudana, R., Ananthula, H. K., Parenky, A. \& Mitra, A. K. Ocular Drug Delivery. AAPS J. 12, 348-360 (2010).

Wu, C., Jiang, P., Li, W., Guo, H., Wang, J., Chen, J., Prausnitz, M. R. \& Wang, Z. L. SelfPowered Iontophoretic Transdermal Drug Delivery System Driven and Regulated by Biomechanical Motions. Adv. Funct. Mater. 30, 1907378 (2020). 
H. M., Chen, K., Shahpar, Z., Talebi, S., Milla, C., Javey, A. \& Davis, R. W. Autonomous sweat extraction and analysis applied to cystic fibrosis and glucose monitoring using a fully integrated wearable platform. Proc. Natl. Acad. Sci. U. S. A. 114, 4625-4630 (2017).

Behar-Cohen, F. F., El Aouni, A., Gautier, S., David, G., Davis, J., Chapon, P. \& Parel, J. M. Transscleral Coulomb-controlled iontophoresis of methyl prednisolone into the rabbit eye: Influence of duration of treatment, current intensity and drug concentration on ocular tissue and fluid levels. Exp. Eye Res. 74, 51-59 (2002).

49 Maulvi, F. A., Lakdawala, D. H., Shaikh, A. A., Desai, A. R., Choksi, H. H., Vaidya, R. J., Ranch, K. M., Koli, A. R., Vyas, B. A. \& Shah, D. O. In vitro and in vivo evaluation of novel implantation technology in hydrogel contact lenses for controlled drug delivery. J. Control. Release 226, 47-56 (2016).

Griffiths, D. J. Introduction to Electrodynamics. (Prentice Hall, 1999).

\section{Acknowledgements}

The authors would like to acknowledge financial support from the National Natural Science Foundation of China (Grant No. 61771498, 61901535, 81970778) and Science and Technology Planning Project of Guangdong Province for Industrial Applications (Grant No. 2017B090917001), Guangdong Province Key Area R\&D Program (Grant No.2018B030332001), Science and Technology Program of Guangzhou, China (Grant No. 202102080192) and Guangdong Basic and Applied Basic Research Foundation (Grant No. 2021A1515012261, 2019A1515012087, 2020A1515010987, 2020A1515110424), Key Program of Sun Yat-Sen University (201gzd14).

\section{Author contributions}

C.Y. and X.X. conceived the concept, designed the work, analysed data and wrote the manuscript. C.Y., Q.N.W., J.Q.L., J.S.M., X.L.L., C.D.Y., Z.Q.L., J.B.Y., L.L.J., W.R.C., H.J.C., J.W., and X.X. performed statistical analyses of datasets and aided in the preparation of displays communicating datasets. X.X. supervised the study. All authors discussed the results and assisted in the preparation of the manuscript. 


\section{Figures}

(a)

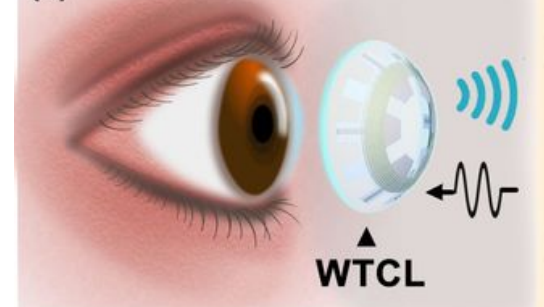

(c) IOP sensing

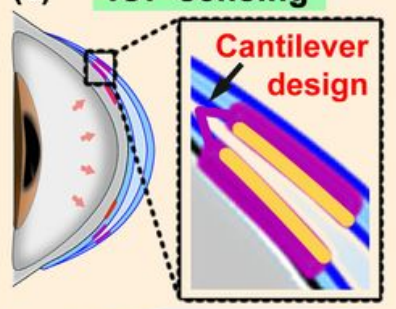

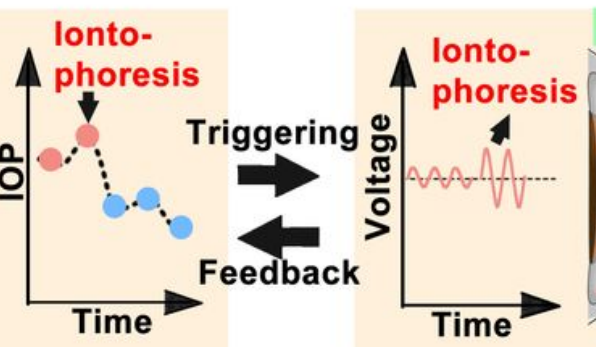

Drug delivery

(b)

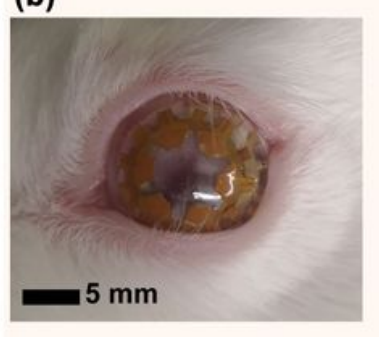

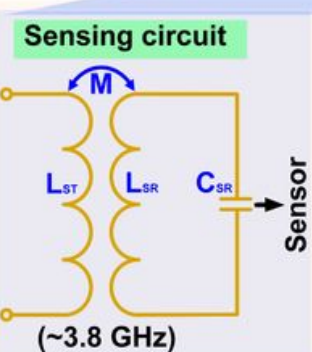

( $\sim 3.8 \mathrm{GHz})$
IOP sensing

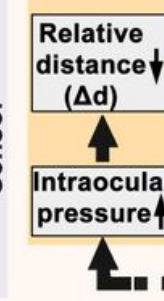

\section{sensing}

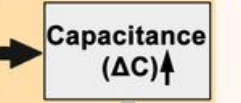

(d)
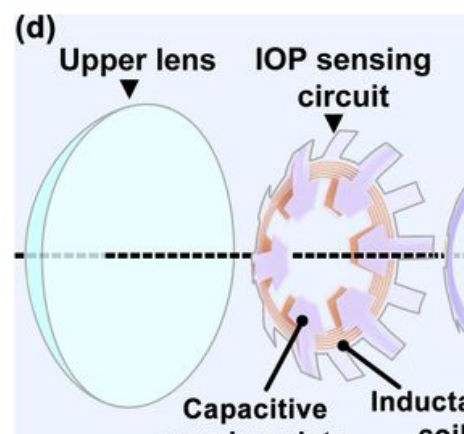

capacive circuit

WPT

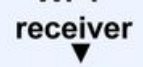

Lower lens lontophoresis electrodes

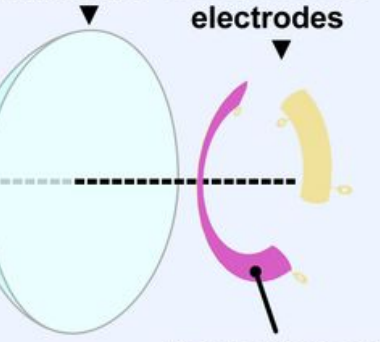

Drugs/Hydrogel

\section{Advantages}

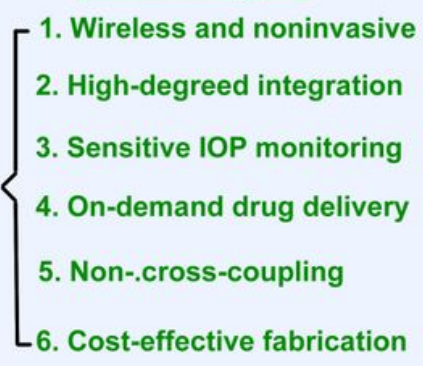

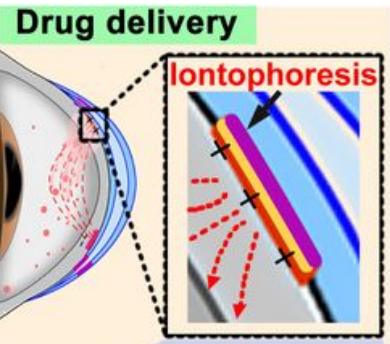

Delivery circuit

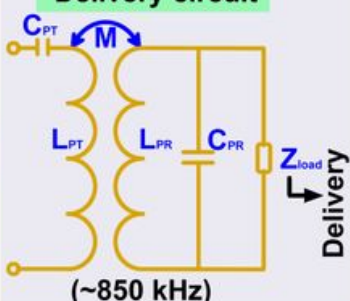

( $850 \mathrm{kHz})$

(e)

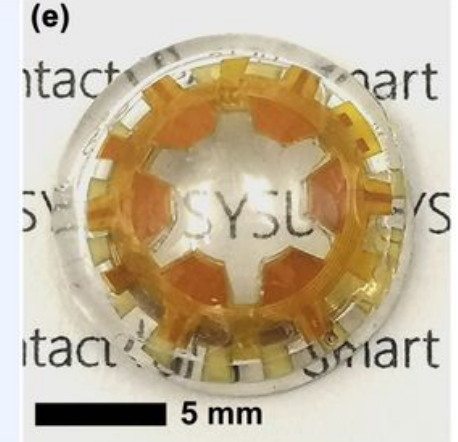

\section{Figure 1}

Schematic of the WTCL for real-time and in situ glaucoma diagnosis and therapy in a closed-loop manner. (a) Schematic of the WTCL for wireless glaucoma diagnosis and therapy. (b) Photograph of WTCL worn on the eyes of a live rabbit. (c) Schematic of wireless operation for the purpose of IOP monitoring and on-demand medicines administration in a closed-loop manner. The soft device, engineered as a double layer contact lens structure, was integrated with an LCR and a WPT receiver circuit. These modules were wirelessly connected to external integrated antenna that could record the IOP signal and trigger iontophoresis for drug delivery if needed. Insert figures respectively highlight critical IOP sensing and drug delivery unit. (d) Structure of the WTCL in an exploded view. (e) Optical image of the WTCL. 
(a)

Layout of IOP sensing circuit

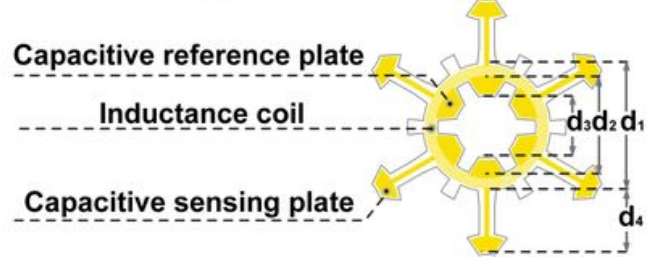

(e) Integrated antenna (f) (I)

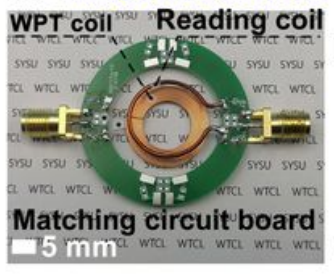
Delivery electrode

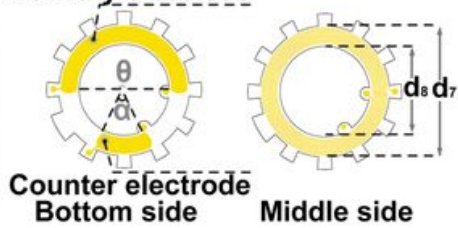

(b)

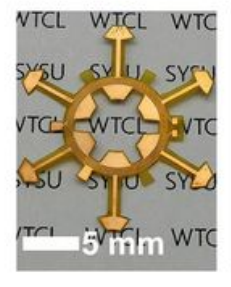

d.

Layout of drug delivery circuit

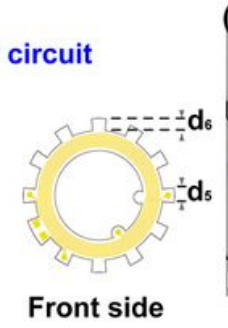

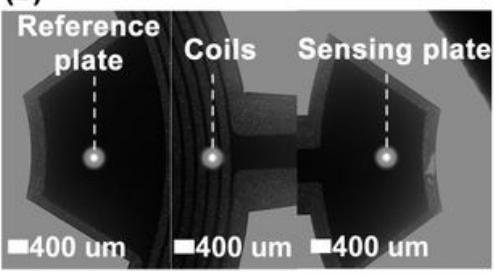

(II)

(III)

B'Attom sidTe' Front side

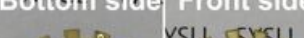

(c)

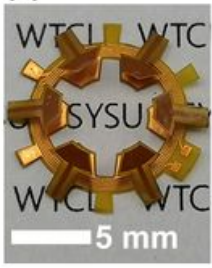

(IV) (d)

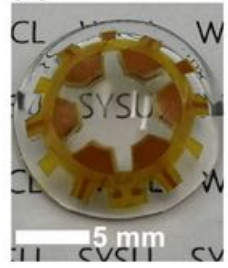

(g)

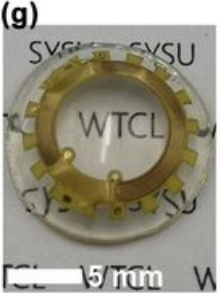

(h) IOP monitoring circuit

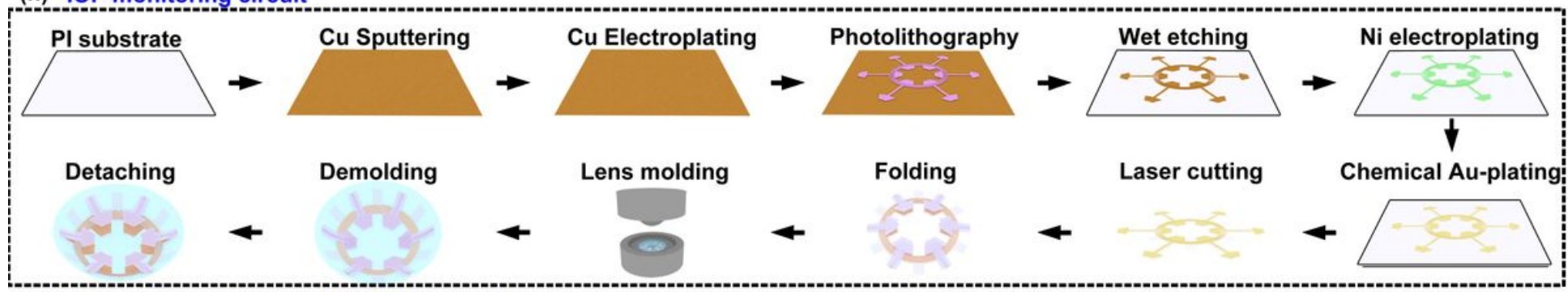

(i) Drug delivery circuit and WTCL integration

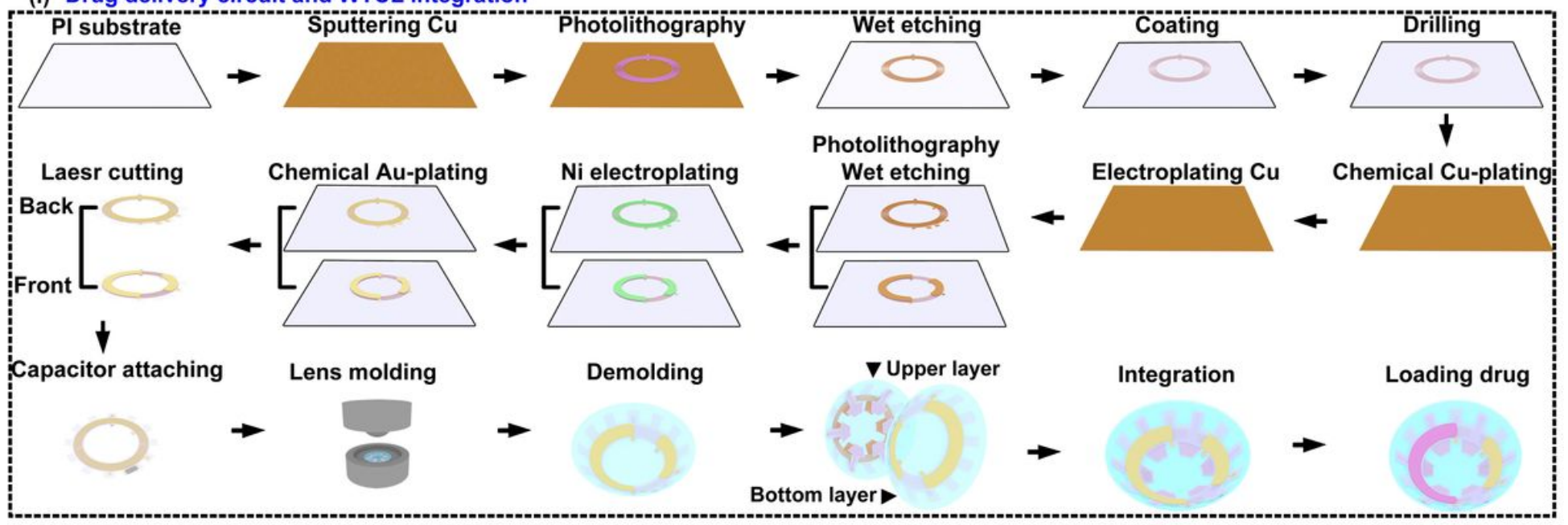

Figure 2

Schematic illustration of the WTCL's design and fabrication process. (a) The snowflake-shaped layout design and the photograph of the sensing circuit. (b) The microscopic image of the reference plate, coils, and sensing plate deployed on sensing circuit. The photograph of (c) the folded sensing circuit and (d) the upper layer of contact lens. (e) Image of the integrated antenna. (f)(I) The flower-shaped layout design, (II) back surface (III) front surface images, and (IV) microscopic image of the drug delivery circuit. (g) The photograph of bottom layer lens integrated with drug delivery circuit. Illustration of the fabrication process of (h) IOP monitoring circuit, (i) drug delivery circuit and the device integration. The fabrication of the sensing and delivery modulus employed a printed circuit process coupled with cast-molding method. 
(a)(I)

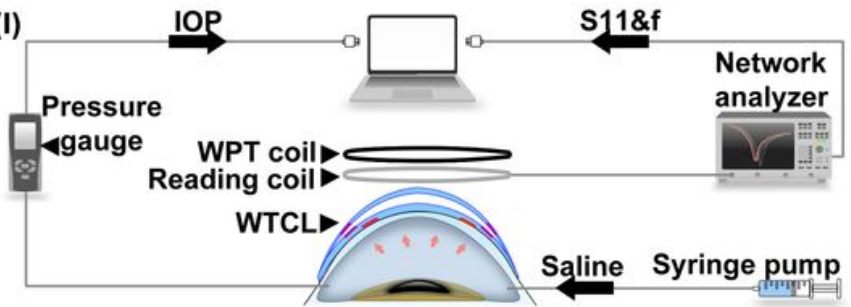

(II)

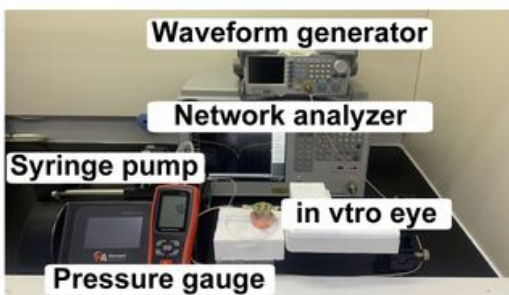

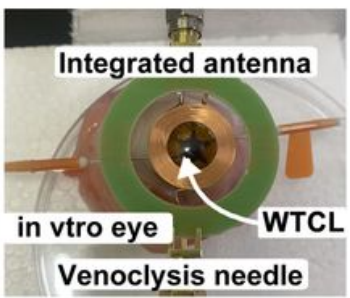

Venoclysis needle

(b) $\quad 5.0 \mathrm{mmHg} \quad 10.0 \mathrm{mmHg} \quad 15.0 \mathrm{mmHg} \quad 20.0 \mathrm{mmHg} \quad 25.0 \mathrm{mmHg} \quad 30.0 \mathrm{mmHg} \quad 35.0 \mathrm{mmHg} \quad 40.0 \mathrm{mmHg} \quad 45.0 \mathrm{mmHg} \quad 50.0 \mathrm{mmHg}$

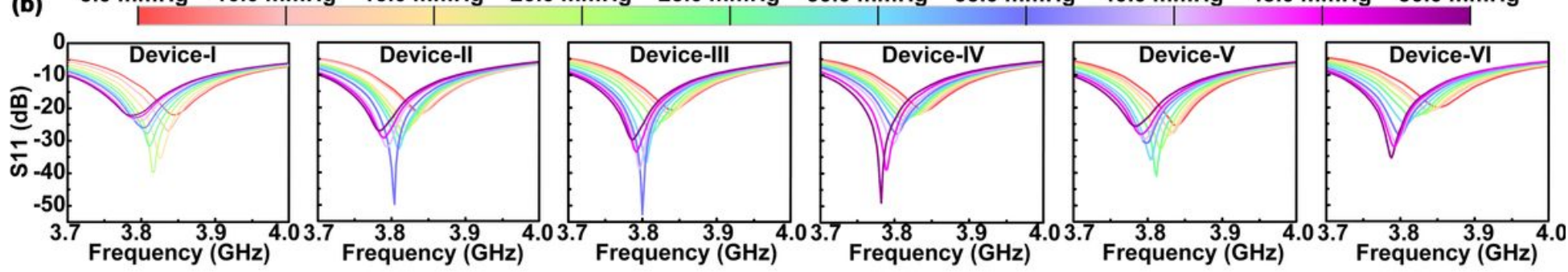

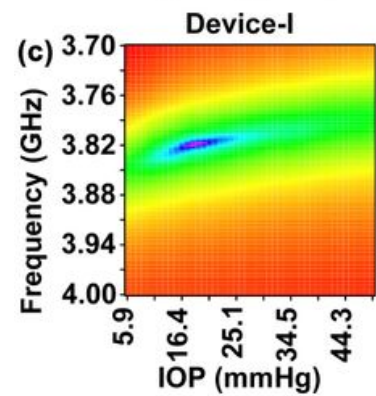
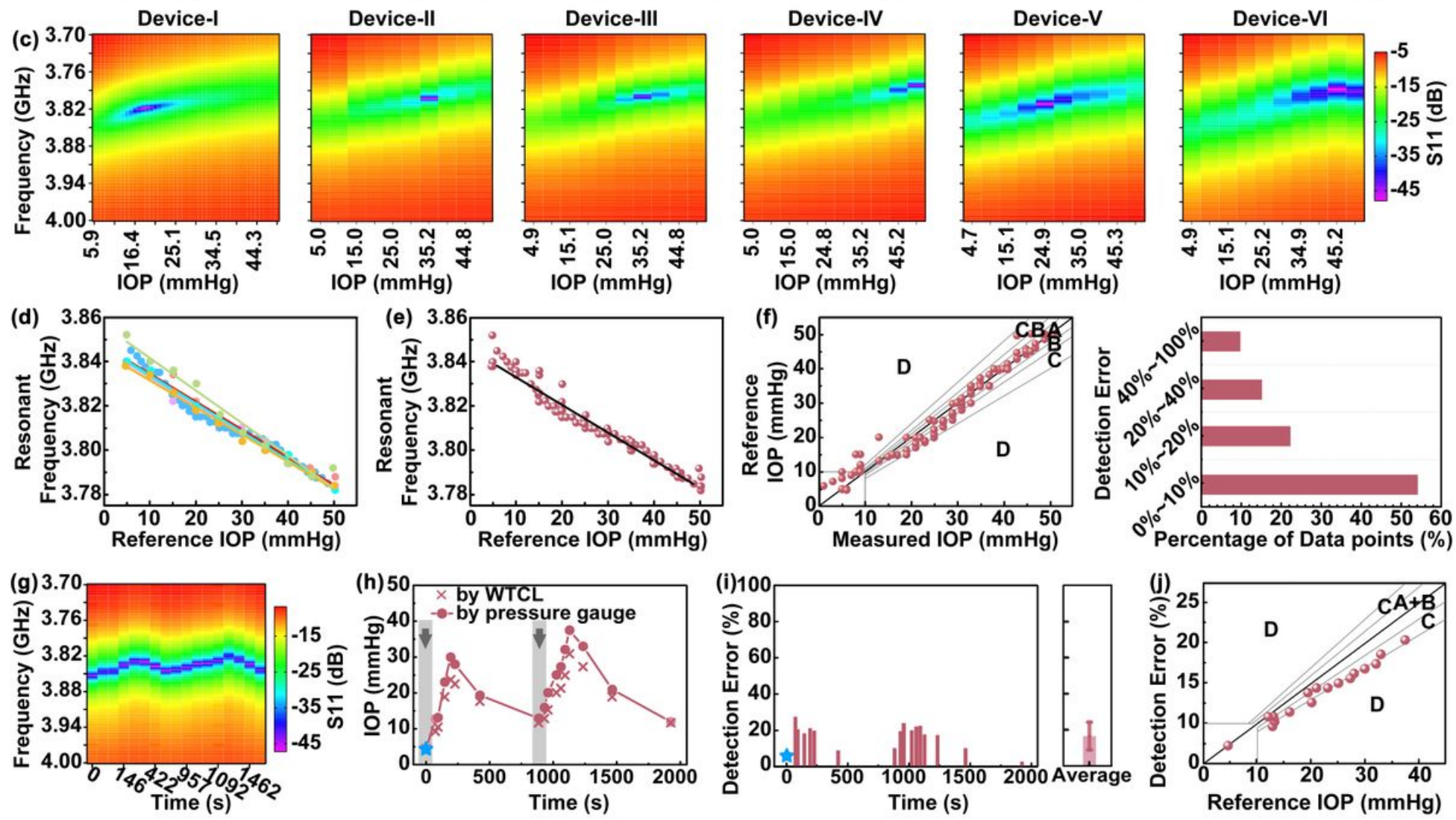

(h)

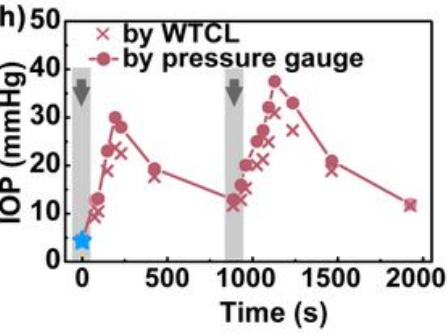

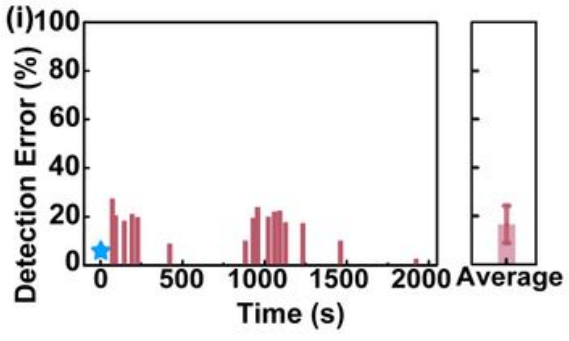

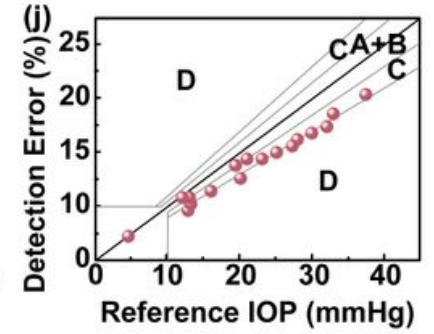

\section{Figure 3}

IOP sensing performance of the WTCL. (a-I) Schematic and (II) experimental set-up of the wireless IOP sensing experiments. (b) The reflection spectra of six representative WTCL devices worn on porcine eyeball at different IOP. (c) The results of the S11 values at different frequencies and IOP conditions in (b) were plotted as heatmap diagram, where the value of S11 exhibited a linear pattern in the frequency-IOP heatmap. (d) Linear regression of resonant frequency versus IOP value of each WTCL device. (e) The averaged linear regression of resonant frequency versus IOP value of the six WTCL devices in (b). (f) Error grid analysis and statical analysis of the IOP sensing accuracy via WTCL. Region A, B, C and D referred to errors $<10 \%, 10-20 \%, 20-40 \%$ and $>40 \%$, respectively. (g) Heatmap plot of the reflection coefficients 
recorded during the continuous recording of IOP via WTCL. (h) Continuous IOP signals monitored by WTCL on in-vitro porcine eyeball. Calibration point using reference IOP was marked with blue asterisks. The black arrow referred to the time point of saline injections. (i) Statistical analysis of detection errors via WTCL compared to commercial pressure gauge at different time points. Calibration point was marked with blue asterisks. (j) Error grid analysis of the continuous IOP sensing via WTCL. Region A+B, C and D referred to errors $<20 \%, 20-40 \%$ and $>40 \%$, respectively.
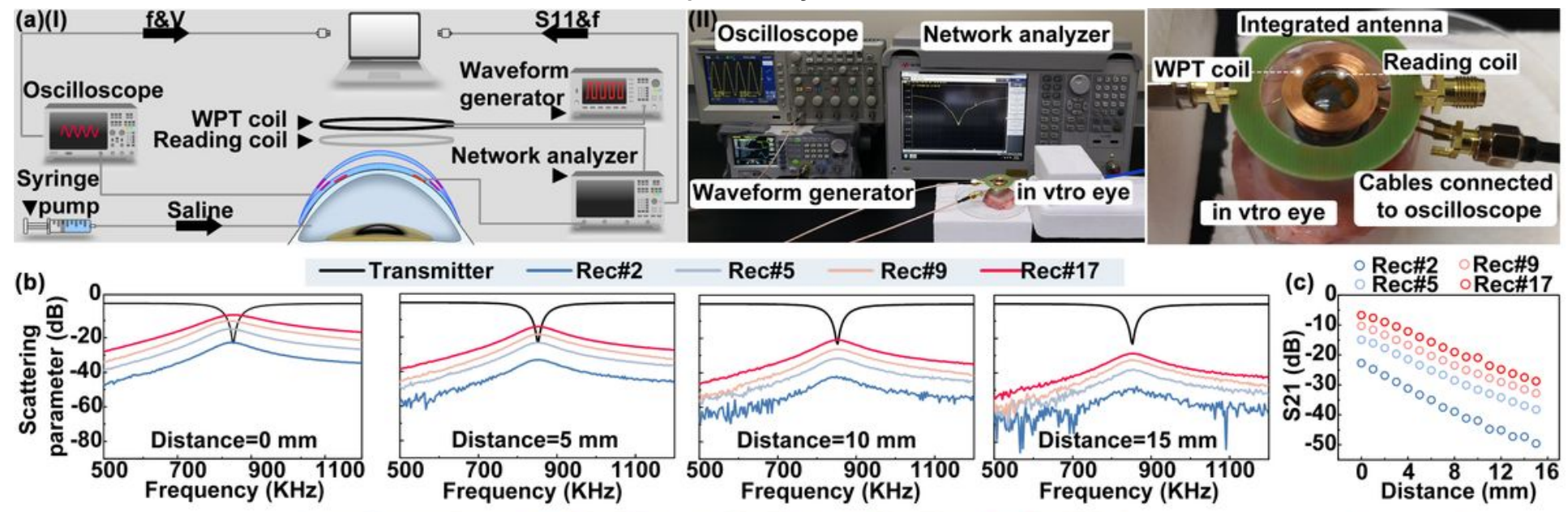

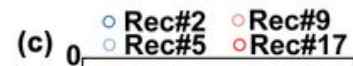
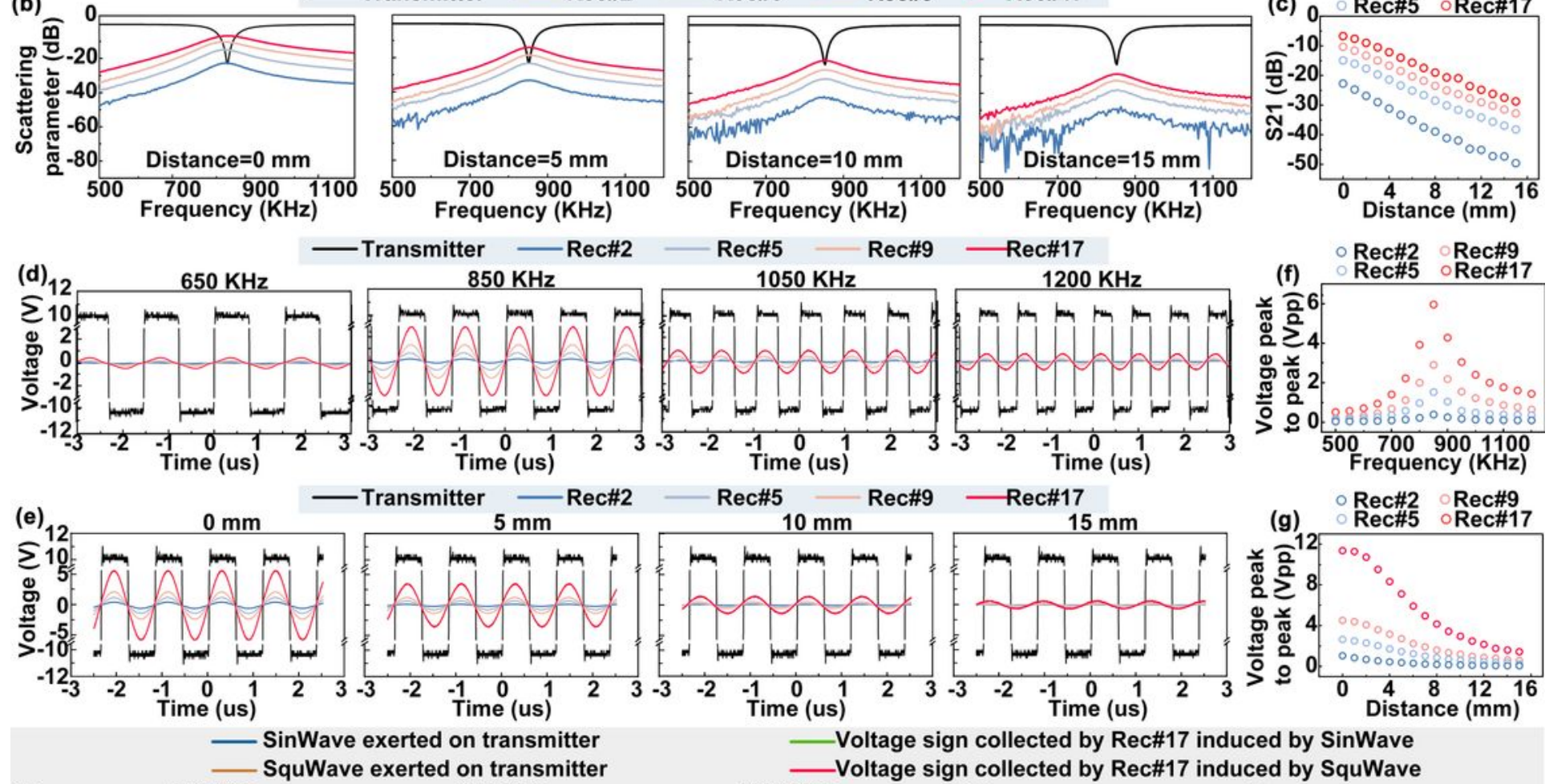

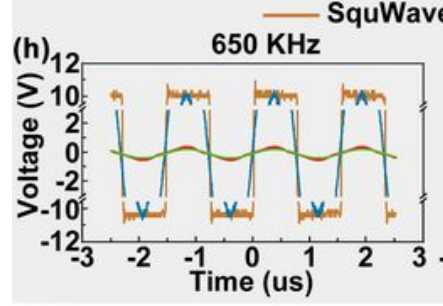

(k)(I)

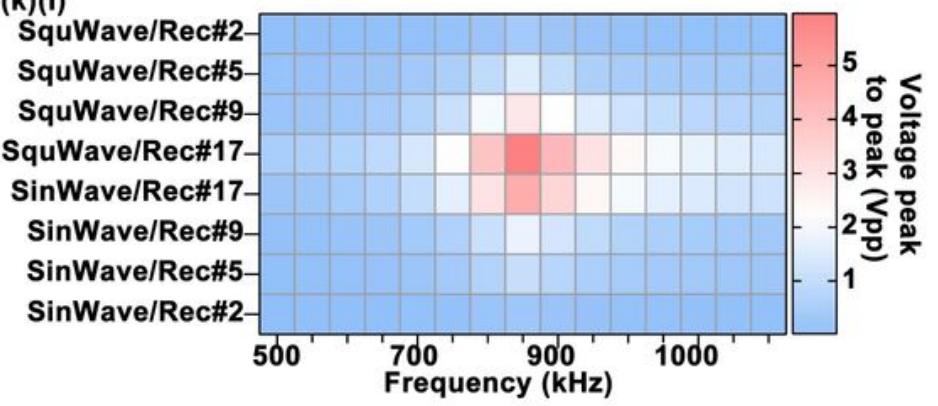

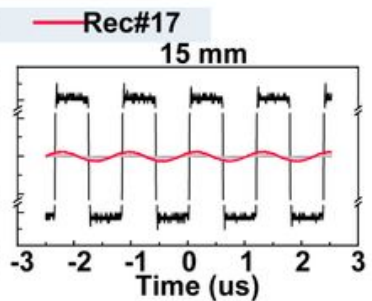

Voltage sign collected by Rec\#17 induced by SinWave

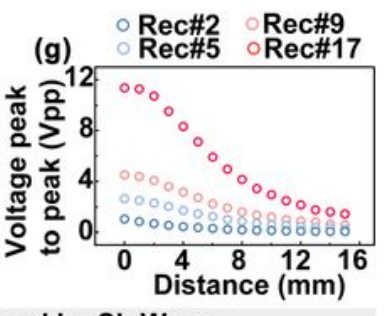

Voltage sign collected by Rec\#17 induced by SquWave
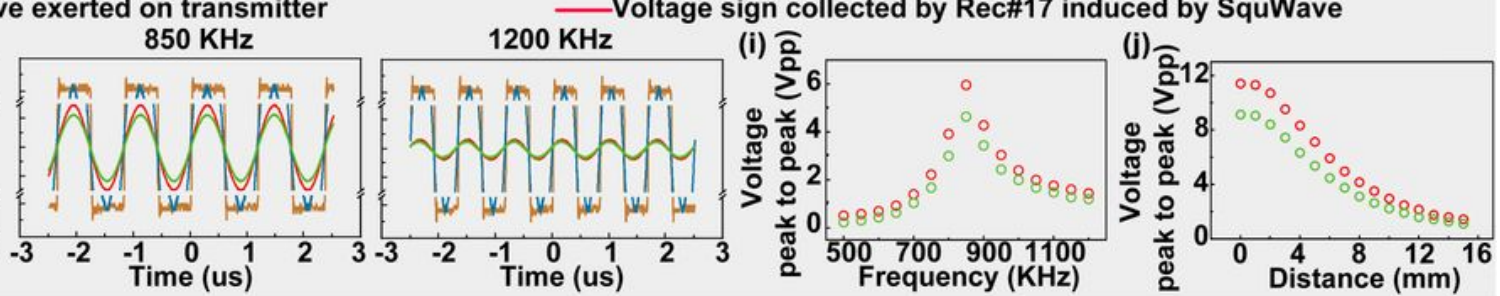

(II)

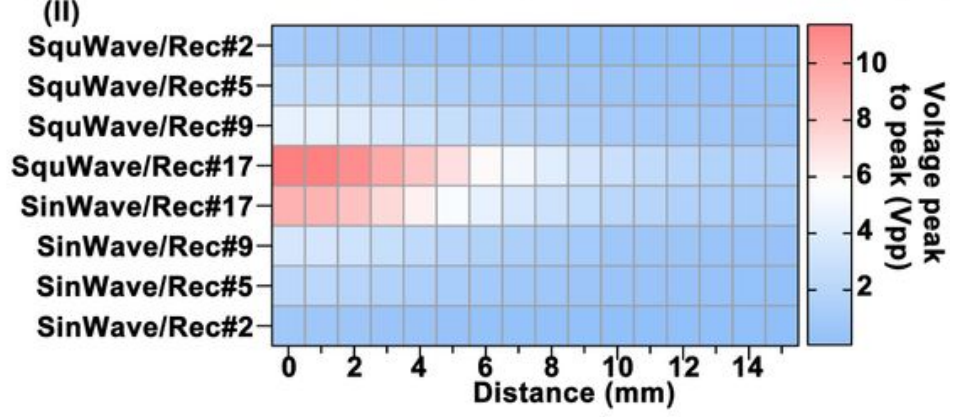


WPT performance of the WTCL. (a-I) Schematic and (II) experimental set-up of the WPT experiments. (b) Reflection coefficient spectra (S11 and S21) recorded from four receivers at different radiation distance. (c) The S21 recorded by the four receivers at $850 \mathrm{kHz}$ were plotted as a function of radiation distance. (d) Alternating voltage signals collected from four receivers wirelessly under different frequency radiation at 20 Vpp applied on transmitter, and (e) Wirelessly transferred alternating voltage waveforms of four receivers under different radiation distance at $20 \mathrm{Vpp}$ applied on transmitter, and (f) the Vpp were plotted as a function of frequency and (g) as a function of distance. (h) The wirelessly transferred voltage signals collected by Rec\#17 activated by SquWave or SinWave voltages at different frequencies, and the Vpp were plotted as a function of (i)frequency or (j) distance. (k) Heatmap plot summarized the Vpp recorded from four receive circuits under different voltage transfer conditions, including the coupling frequency, the radiation distance, and the waveforms.

(a)

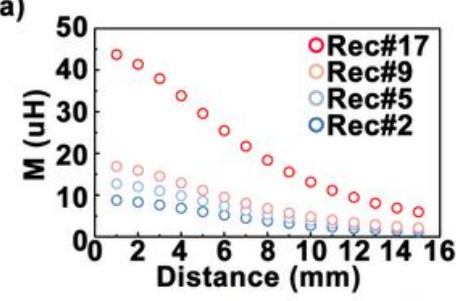

(b)

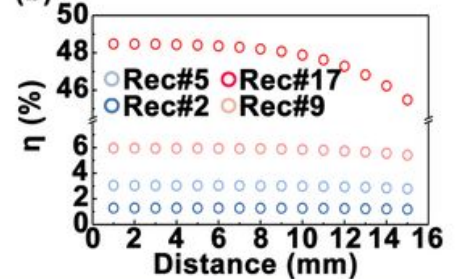

(c)

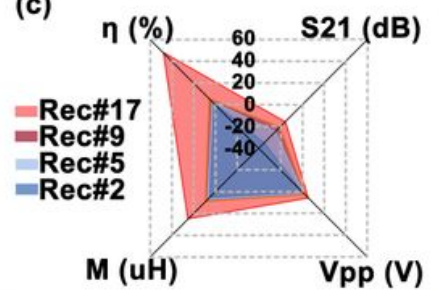

(f)

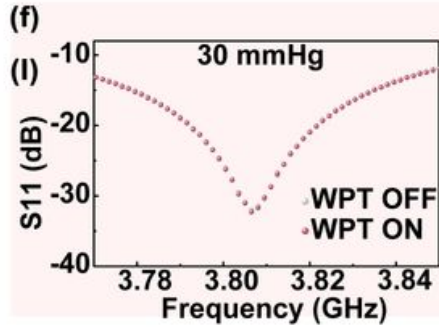

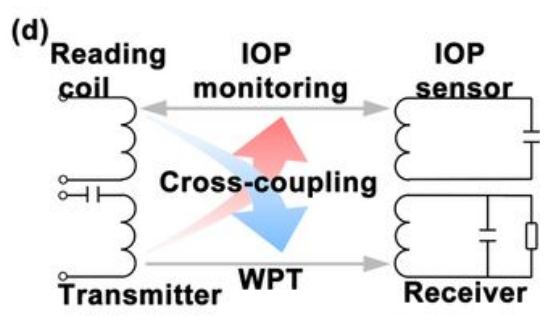
S11-OFF- Resonant frequency-OFF
- S11-ON - Resonant frequency-ON

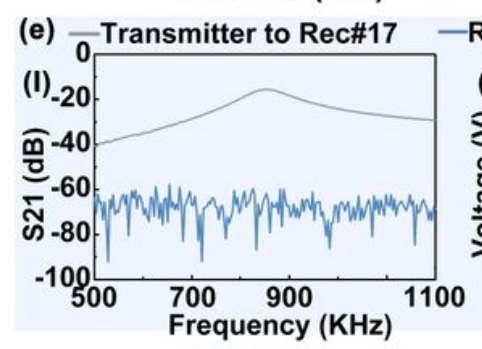

(g)

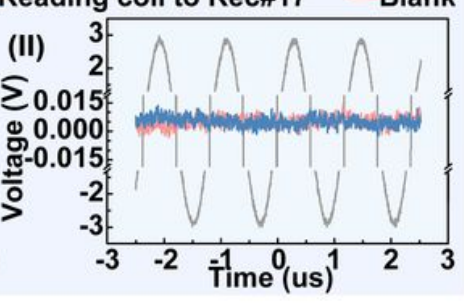

(h)
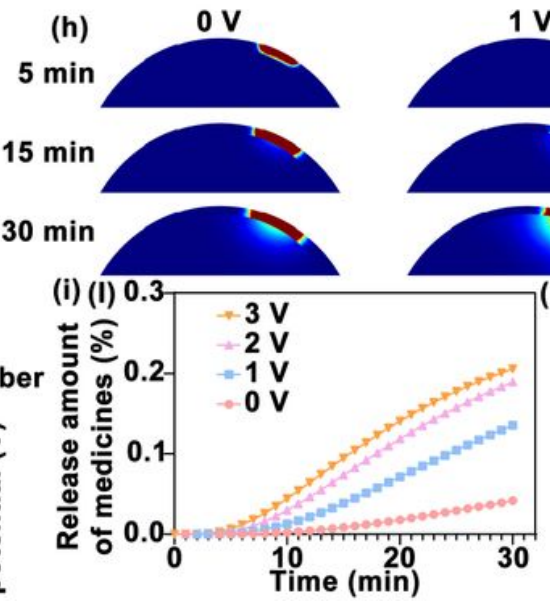

$1 \mathrm{~V}$
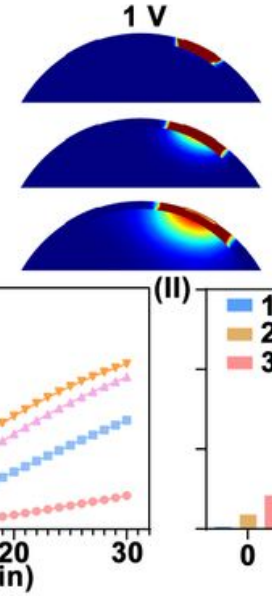
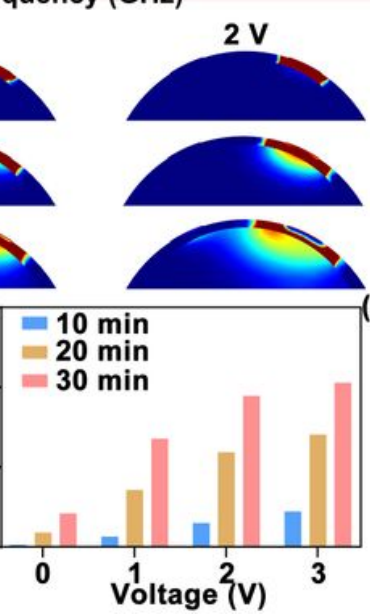

(III)
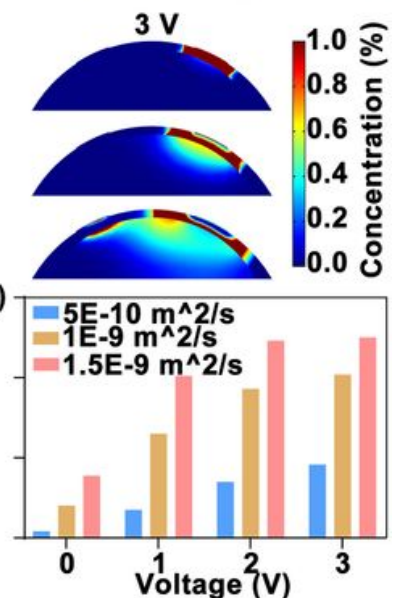

Figure 5

(a) The mutual inductance and (b) Power transfer efficiency were theoretically calculated according to the circuit design of four receivers and the radiation distance. (c) Rader chart summarized performance (S21, Vpp, M, and $\eta$ ) of etch WPT group (Rec\#2, Rec\#5, Rec\#9 or Rec\#17 linked to transmitter with 6 mm radiation distance and $850 \mathrm{kHz}$ ) (d) Schematic showing the experiments studying the cross-coupling between IOP monitoring and WPT module. Red arrow denoted the interference generated by the radiation of WPT transmitter to sensing module. Blue arrow denotes the cross-coupling between IOP reading coil 
and the WPT receiver. All examinations were performed with the radiation distance of $6 \mathrm{~mm}$. (e) The IOP reading coil and WPT transmitter were coupled with the WPT receiver (using Rec\#17), respectively, and the S21 indicating the coupling efficiency and the generated voltages on receiver radiated at $850 \mathrm{kHz}$ were separately measured. (f) The WTCL was placed on porcine eye at different IOP, and the reading signals (the resonance frequency and the S11) were recorded with or without the presence of radiation from WPT transmitter. (g) The (top) 3D COMSOL model and (bottom) the simulated distribution profile of electric potential and electric field through the anterior region under the condition of iontophoresis at $3 \mathrm{~V}$ for $\mathrm{t}=30 \mathrm{~min}$. (h) The time slots of drug concentration profile delivered by WTCL at various applied voltages $(0,1,2$, and $3 \mathrm{~V})$. (i) The delivered amounts of drugs at different conditions, including the (I) applied voltages, (II) iontophoretic duration and (III) assumed diffusivities, were systematically evaluated. 


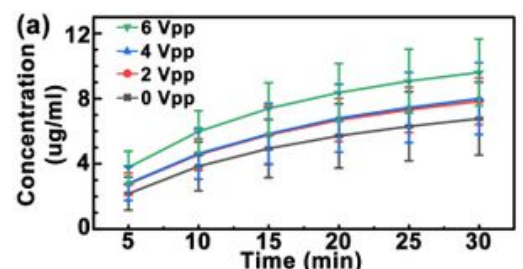

(b)
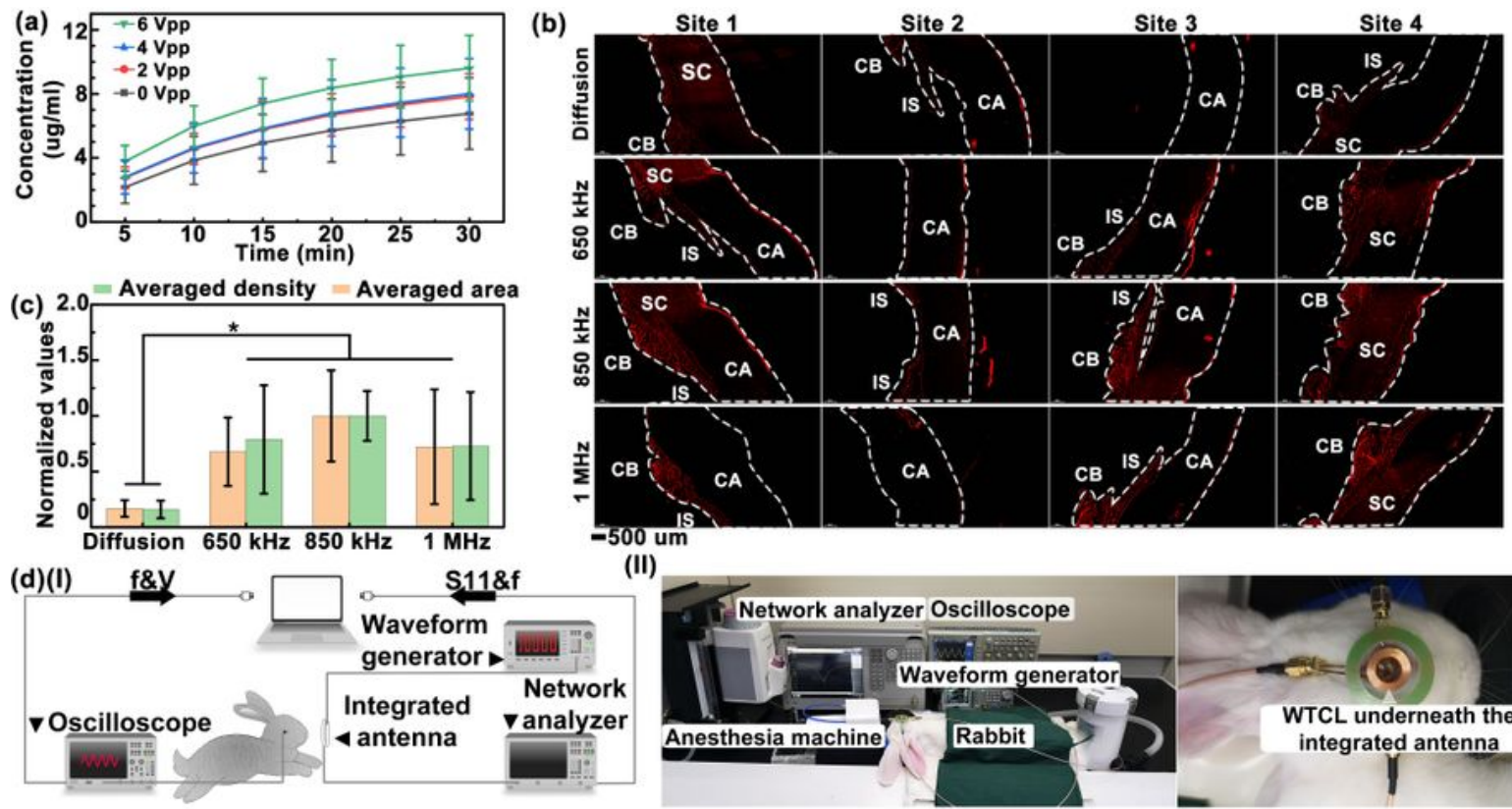

(II)
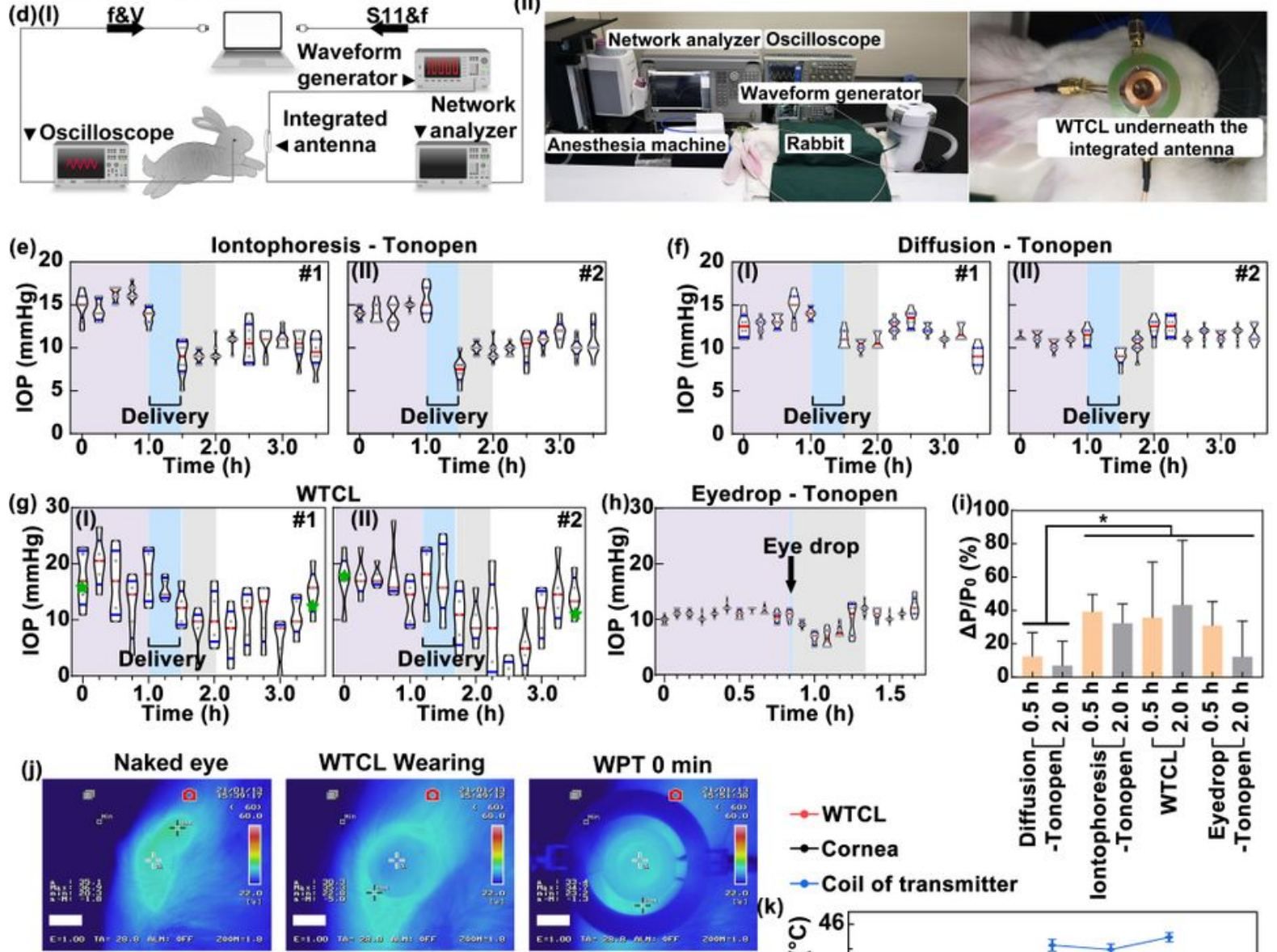

WTCL Wearing
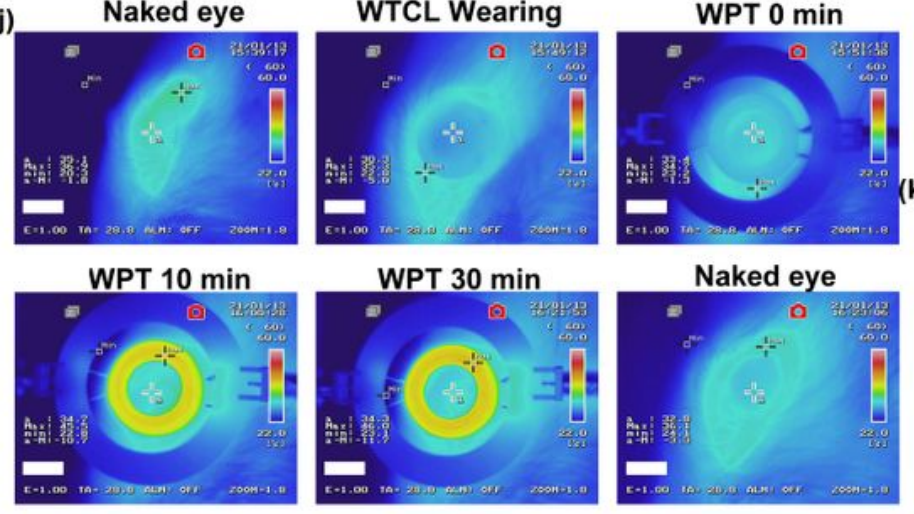

$\rightarrow$ WTCL

$\rightarrow$-Cornea

$\rightarrow$ Coil of transmitter
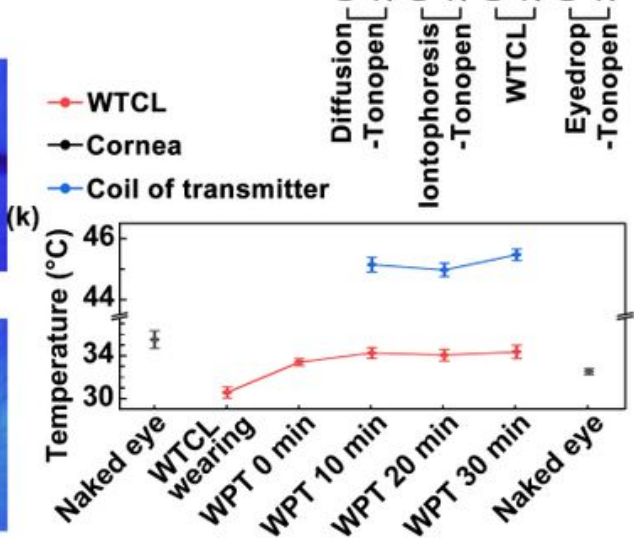

Figure 6

Sensing and therapeutic performance of the integrated WTCL. (a) Quantitative analysis of in vitro rhodamine $B$ released from WTCL at different alternating voltages for 30 min. $N=6$ measurements. (b) Rhodamine $B$ was utilized as the medicines analog in ex vivo experiments on porcine eyes, to examine the influence of iontophoresis on drug delivery across cornea. CB, ciliary body; IS, iris; CA, Cornea. Scale bar is $500 \mu \mathrm{m}$. After delivery, fluorescence visualization in the anterior tissue was observed via 
microscope and (c) quantitatively analyzed. $\mathrm{N}=4$ sites per group. (d-I) Schematic and (II) experimental setup of the in vivo WTCL experiments. The rabbits were anesthetized and worn with WTCL on their eyes, while the signals recording of WTCL and WPT operation were conducted by the integrated antenna. The rabbits' IOP were monitored with Tonopen, and brimonidine deliveries were performed via (e) Wirelessly powered iontophoresis or ( $f$ ) passive free diffusion based on WTCL. (g) Simultaneous IOP sensing and drug delivery using a single WTCL device. The rabbit's IOP were wirelessly monitored with the WTCL, and brimonidine delivery via wireless iontophoresis on the same WTCL was conducted. The green asterisk indicated the IOP measurements via Tonopen for calibration or accuracy comparison. (h) Rabbit's eye was treated with eye drops of brimonidine, and the IOP was measured via Tonopen. $\mathrm{N}=2$ rabbits in (e)(g) and $\mathrm{N}=1$ rabbit in (h). In (e)-(h), at each time point, 8 Topopen measurements were conducted, and the purple, blue, gray and white regions indicated the periods of prior to delivery, during delivery, $0.5 \mathrm{~h}$ after delivery and $2 \mathrm{~h}$ after delivery, respectively. (i) The IOP reductions effects after 0.5 and $2 \mathrm{~h}$ of drug delivery via iontophoresis, free diffusion and eye drops were summarized. Data were presented as mean \pm s.d. Significance was evaluated by one-way analysis of variance. ${ }^{*} P<0.05$. (j) Monitoring of the thermal effects generated during WPT operation via infrared thermal camera, and $(k)$ the temperate on cornea, WTCL, and transmitter coils during WPT process were analyzed. $\mathrm{N}=4$ measurements per group. Data were presented as mean \pm s.d. Significance was evaluated by one-way analysis of variance. ${ }^{*} \mathrm{P}<$ 0.05 .

\section{Supplementary Files}

This is a list of supplementary files associated with this preprint. Click to download.

- SupplementaryMaterials.docx 